\title{
LIMITS ON OPTICAL POLARIZATION DURING THE PROMPT PHASE OF GRB 140430A
}

\author{
D. KopaČ ${ }^{1,2}$, C. G. Mundell ${ }^{1,3}$, J. Japelj ${ }^{2}$, D. M. Arnold ${ }^{1}$, I. A. Steele ${ }^{1}$, C. Guidorzi ${ }^{4}$, S. Dichiara ${ }^{4}$, S. Kobayashi ${ }^{1}$, \\ A. Gomboc ${ }^{2}$, R. M. Harrison ${ }^{5}$, G. P. Lamb ${ }^{1}$, A. Melandri ${ }^{6}$, R. J. Smith ${ }^{1}$, F. J. Virgili ${ }^{1}$, A. J. Castro-Tirado ${ }^{7,8}$, \\ J. Gorosabel ${ }^{7,9,12}$, A. Järvinen ${ }^{10}$, R. SÁnCheZ-RAmírez ${ }^{7}$, S. R. OATEs ${ }^{7}$, ANd M. JelíneK ${ }^{11}$ \\ ${ }^{1}$ Astrophysics Research Institute, Liverpool John Moores University, 146 Brownlow Hill, Liverpool, L3 5RF, UK; drejc.kopac@ @mf.uni-lj.si \\ ${ }^{2}$ Department of Physics, Faculty of Mathematics and Physics, University of Ljubljana, Jadranska 19, 1000 Ljubljana, Slovenia \\ ${ }^{3}$ Department of Physics, University of Bath, Claverton Down, Bath, BA2 7AY, UK \\ ${ }^{4}$ Department of Physics and Earth Sciences, University of Ferrara, via Saragat 1, I-44122, Ferrara, Italy \\ 5 Department of Astrophysics, School of Physics and Astronomy, Tel Aviv University, 69978 Tel Aviv, Israel \\ ${ }^{6}$ INAF-Osservatorio Astronomico di Brera, via E. Bianchi 46, I-23807 Merate (LC), Italy \\ ${ }^{7}$ Instituto de Astrofísica de Andalucía (IAA-CSIC), Glorieta de la Astronomia s/n, E-18008 Granada, Spain \\ ${ }^{8}$ Departamento de Ingeniaría de Sistemas y Automática, E.T.S.I. Industriales, Universidad de Málaga, C/. Doctor Ortiz Ramos s/n, \\ Campus de Teatinos, E-29071 Málaga, Spain \\ ${ }^{9}$ Grupo de Ciencias Planetarias, Escuela Superior de Ingenieros, Física Aplicada I, Alameda de Urquijo s/n, E-48013 Bilbao, Spain \\ ${ }^{10}$ AIP-Leibniz-Institut für Astrophysik Potsdam, An der Sternwarte 16, D-14482 Potsdam, Germany \\ ${ }^{11}$ ASU-CAS-Astronomical Institute of the Czech Academy of Sciences, Fričova 298, 25165 Ondřejov, Czech Republic \\ Received 2015 June 17; accepted 2015 September 9; published 2015 October 21
}

\begin{abstract}
Gamma-ray burst GRB 140430A was detected by the Swift satellite and observed promptly with the imaging polarimeter RINGO3 mounted on the Liverpool Telescope, with observations beginning while the prompt $\gamma$-ray emission was still ongoing. In this paper, we present densely sampled (10-s temporal resolution) early optical light curves (LCs) in 3 optical bands and limits to the degree of optical polarization. We compare optical, X-ray, and gammaray properties and present an analysis of the optical emission during a period of high-energy flaring. The complex optical LC cannot be explained merely with a combination of forward and reverse shock emission from a standard external shock, implying additional contribution of emission from internal shock dissipation. We estimate an upper limit for time averaged optical polarization during the prompt phase to be as low as $P<12 \% \quad(1 \sigma)$. This suggests that the optical flares and early afterglow emission in this GRB are not highly polarized. Alternatively, time averaging could mask the presence of otherwise polarized components of distinct origin at different polarization position angles.
\end{abstract}

Key words: gamma-ray burst: general - gamma-ray burst: individual (GRB 140430A) instrumentation: polarimeters

\section{INTRODUCTION}

Gamma-ray bursts (GRBs) are powerful cosmic explosions, first identified by the detection of short flashes of gamma-ray emission by military satellites in the 1960s (Klebesadel et al. 1973) and, today, are thought to represent the end product of massive stellar core-collapse or the merger of compact objects (e.g., Mészáros 2006; Vedrenne \& Atteia 2009; Gomboc 2012). In addition to being interesting in their own right—as black holes: jet systems with ultra-relativistic expansion speeds and potentially strong magnetic fieldsGRBs are also among the most distant known objects in the universe and thus act as probes of the early universe (Salvaterra et al. 2009; Tanvir et al. 2009; Cucchiara et al. 2011).

In the standard fireball model of GRBs (e.g., Piran 1999), prompt gamma-ray emission is produced by internal shocks (Rees \& Mészáros 1994), and the long-lasting afterglow emission at longer wavelengths is produced by external shocks when relativistic ejecta collide and are decelerated by the surrounding circumburst material (Rees \& Mészáros 1992; Mészáros \& Rees 1997). Despite the overall success of this framework, the prompt emission mechanism is still poorly known, and internal shocks remain an inefficient mechanism for the conversion of kinetic to radiated energy (e.g., Beloborodov 2005; Rees \& Mészáros 2005; Zhang \& Yan 2011; Axelsson \& Borgonovo 2015; Beniamini et al. 2015). In

\footnotetext{
${ }^{12}$ Deceased April 2015.
}

addition, although the generally accepted external shock model works well for smoothly fading late time ( days post burst) afterglows, observations of early afterglow light curves (LCs) in the first minutes to hours after the burst, especially in the era of the Swift satellite (Gehrels et al. 2004), show an unexpected wealth of variety, attributed to a range of mechanisms including internal and external shocks, long-lived central engines and double jet structures (e.g., Monfardini et al. 2006; Mundell et al. 2007b; Gomboc et al. 2008; Melandri et al. 2008, 2010; Kopač et al. 2013; Virgili et al. 2013; Japelj et al. 2014; de Pasquale et al. 2015).

Bursts with longer-lasting prompt emission or those with very bright optical afterglows - detectable by small telescopes with wide-fields of view-have provided the best chance to detect longer wavelength emission during the prompt phase. Although the sample is still relatively small, an increasing number of flares at wavelengths below the gamma-ray bands have been detected. ${ }^{13}$ Similar observations have also been obtained for X-ray flashes. ${ }^{14}$

\footnotetext{
${ }^{13}$ For a discussion of X-ray flares, see, e.g., Burrows et al. (2005), O’Brien et al. (2006), Chincarini et al. (2007), and Margutti et al. (2010), and for optical flares/rebrightenings, see, e.g., Akerlof et al. (1999), Blake et al. (2005), Vestrand et al. (2005), Page et al. (2007), Mundell et al. (2007a), Racusin et al. (2008), Thöne et al. (2010), Guidorzi et al. (2011), Gendre et al. (2012, 2013), Kopač et al. (2013), Virgili et al. (2013), Elliott et al. (2014), Greiner et al. (2014), Vestrand et al. (2014), and Nappo et al. (2014).

14 e.g., Boër et al. (2006), Krühler et al. (2009), and Guidorzi et al. (2009).
} 
The origin and connection between different observed spectral components in the prompt phase remains problematic. Prompt GRB LCs often show rapid variability, which can be explained within internal shock dissipation models (Kobayashi et al. 1997). Alternative scenarios have been suggested, such as inverse-Compton scattering (e.g., Panaitescu 2008), large-angle emission (e.g., Kumar \& Panaitescu 2008), structured outflow (e.g., Panaitescu \& Vestrand 2008), anisotropic emission (e.g., Beloborodov et al. 2011), magnetic reconnection (e.g., Zhang \& Yan 2011), etc. In particular, the study of early time optical emission and its polarization properties has provided valuable insight into GRB emission mechanisms (e.g., Yost et al. 2007a, 2007b; Kopač et al. 2013), jet composition, and magnetic field properties (e.g., Mundell et al. 2007a, 2013; Gomboc et al. 2009; Steele et al. 2009; Japelj et al. 2014; King et al. 2014), but observational limitations remain major complications. Specifically, the prompt optical emission is, in most cases, relatively faint and, due to this, temporal resolution in the optical band is often inadequate for direct comparison with gamma-ray LCs, resulting in losing information on intrinsic complexity which imprints the central engine behavior. In short, observing and understanding the prompt and early afterglow emission of GRBs remains technically and theoretically challenging. Therefore, GRBs for which multiwavelength data is obtained simultaneously with the prompt gamma-ray emission and at high temporal cadence are of particular value.

Here we present exquisitely sampled three-band simultaneous multicolor LCs of long-duration GRB 140430A, observed with the RINGO3 polarimeter (Arnold et al. 2012) on the 2-m robotic Liverpool Telescope (LT; Steele et al. 2004; Guidorzi et al. 2006). The prompt gamma-ray emission lasted for $200 \mathrm{~s}$, with fainter emission detected as late as $575 \mathrm{~s}$ after the burst. The optical observations with LT began just $124 \mathrm{~s}$ after the onset of the burst and were contemporaneous with the gamma and X-ray flares at this time. We present the observations and data reduction in Section 2, the analysis of the LCs in Section 3, the discussion of our results in the context of the fireball model in Section 4, and the conclusion in Section 5. Throughout the paper, the convention $F_{\nu}(t) \propto t^{-\alpha} \nu^{-\beta}$ is used to describe the flux density. $\Lambda \mathrm{CDM}$ cosmology is assumed with parameters $H_{0}=67.3 \mathrm{~km} \mathrm{~s}^{-1} \mathrm{Mpc}^{-1}, \Omega_{\Lambda}=0.68, \Omega_{\mathrm{M}}=0.32$ (Planck Collaboration 2014). Best-fit parameters are given at a $1 \sigma$ confidence level, except when stated otherwise. Times are given with respect to the GRB trigger time $t_{0}$.

\section{OBSERVATIONS AND REDUCTION}

\subsection{Swift}

On 2014 April 30, at $t_{0}=20: 33: 36 \mathrm{UT}$, the Burst Alert Telescope (BAT; Barthelmy et al. 2005) on board the Swift satellite triggered on the long GRB 140430A and immediately slewed to the burst (Siegel et al. 2014). The BAT gamma-ray LC shows a multi-peaked structure with two intense peaks: the first one starts at approximately $-10 \mathrm{~s}$ and ends at $\sim 10 \mathrm{~s}$, and the second softer one starts at $\sim 140 \mathrm{~s}$ and ends at $\sim 200 \mathrm{~s}$ post trigger. There are at least two slightly fainter and softer peaks centered at $\sim 25 \mathrm{~s}$ and $\sim 575 \mathrm{~s}$. The $T_{90}(15-350 \mathrm{keV})$ of $174 \pm$ $4 \mathrm{~s}$, fluence $(15-150 \mathrm{keV})$ of $1.1 \pm 0.2 \times 10^{-6} \mathrm{erg} \mathrm{cm}^{-2}$, and time-averaged spectrum power-law index $\Gamma$ of $2.0 \pm 0.2$ (Krimm et al. 2014) put this GRB toward the long-soft end of Swift GRBs (Sakamoto et al. 2011). Fitting the BAT time-averaged spectrum

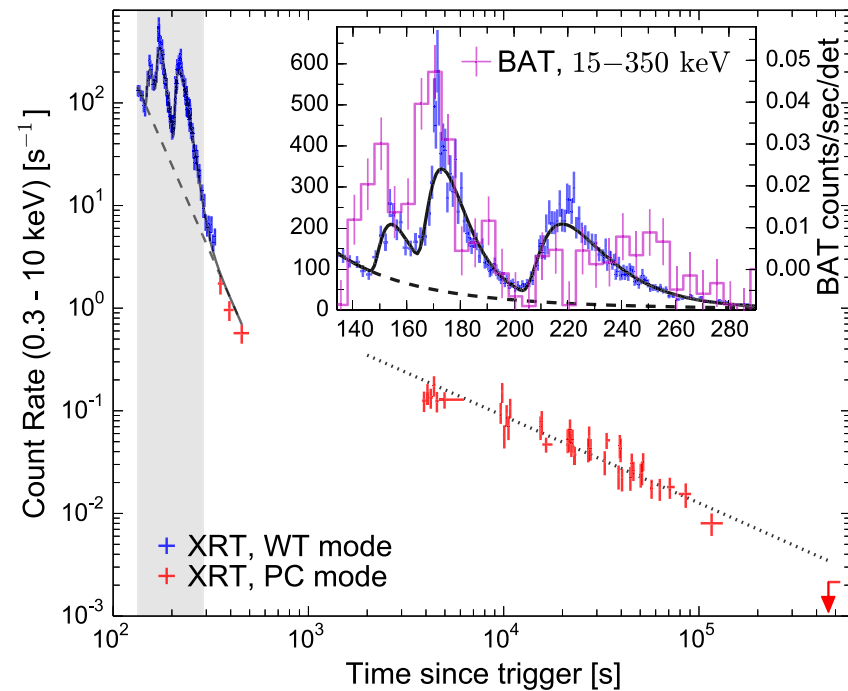

Figure 1. GRB 140430A X-ray light curve obtained from the XRT repository (Evans et al. 2009). The inset plot shows early time behavior in linear scale, together with the gamma-ray light curve (violet histogram, $5 \mathrm{~s}$ uniform binning) obtained from the BAT instrument. Black solid and dash lines represent the fitted model as described in Section 3.1.

with a typical Band function assuming $\alpha=-1$ and $\beta=-2.3$ gives $E_{\text {peak }}^{\text {obs }} \sim 20 \mathrm{keV}$ and, moving to $1-10^{4} \mathrm{keV}$ in the host rest frame at a redshift of $z=1.6$, gives an isotropic-equivalent energy of $E_{\gamma, \text { iso }}=(1.3 \pm 0.4) \times 10^{52} \mathrm{erg}$.

The Swift X-ray Telescope (XRT; Burrows et al. 2005) began follow-up observations at $50.8 \mathrm{~s}$, while gamma-ray emission was still ongoing. A bright and uncataloged fading X-ray source has been detected at R.A.(J2000) $=06^{\mathrm{h}} 51^{\mathrm{m}} 44^{\mathrm{s}} .6$, decl.(J2000) $=+23^{\circ} 01^{\prime} 25^{\prime \prime}$ with an enhanced $90 \%$ confidence uncertainty of 1 ".9 (Evans et al. 2014). The X-ray LC at early times is dominated by at least three bright flares, centered at 154, 171, and $222 \mathrm{~s}$, as seen in Figure 1. The first two flares track the gamma-ray LC both temporally and in brightness (see Figures 1 and 3). The last flare is followed by a steep decay. From $\sim 500$ to $\sim 3900 \mathrm{~s}$ a data gap in X-ray is due to the Earth limb constraint. At later times, the X-ray LC shows a decay until $\sim 10^{5} \mathrm{~s}$.

When transforming the X-ray LC obtained from the XRT LC repository (Evans et al. 2009) from flux to flux density units, we took into account strong spectral evolution at early times and by assuming a power-law spectrum with a given spectral index for each point (see Figure 5), we calculated the flux density at $10 \mathrm{keV}$ by integrating the energy spectrum in the $0.3-10 \mathrm{keV}$ interval. At later times, we instead assumed an average spectral index for every point (see Section 3.1).

The Swift Ultraviolet/Optical Telescope (UVOT; Roming et al. 2005) began observing the GRB field at $183 \mathrm{~s}$ and found a candidate afterglow at the position consistent with the XRT, with estimated magnitude in the UVOT White filter of $18.17 \pm 0.09 \mathrm{mag}$ (Breeveld \& Siegel 2014).

\subsection{Liverpool Telescope}

LT responded to the Swift GRB alert automatically and started observations with the RINGO3 instrument at 20:35:40 UT, i.e., $124 \mathrm{~s}$ after the BAT trigger (Melandri et al. 2014). Fast response to the trigger resulted in obtaining the optical observations contemporaneously with the ongoing 
gamma-ray and X-ray emission during high-energy flares at early times (Figure 3). Observations continued for the next hour, during which the majority of the observations were performed with RINGO3, with a $6 \times 10 \mathrm{~s}$ sequence of IO:O observations with an SDSS $r^{\prime}$ filter inserted at $\sim 33$ minutes to allow for real-time afterglow identification during the observations.

RINGO3 is a novel three-band fast-readout optical imaging polarimeter, which uses a polaroid that rotates once per second. By analyzing relative intensities at eight different orientations of the polaroid, the polarization for each source in the image can be measured, while summing the data from all rotation angles allows derivation of the total flux of each source. Short $125 \mathrm{~ms}$ exposures and zero read-out noise allows optimization of frame co-adding in the data post-processing stages. In addition, a light entering the instrument and passing the rotating polaroid is split into three beams using a pair of dichroic mirrors, and simultaneously imaged using 3 separate EMCCD cameras. The wavelength bands ${ }^{15}$ are determined by dichroics: $V$ ranges from $\sim 350$ to $640 \mathrm{~nm}, R$ ranges from $\sim 650$ to $760 \mathrm{~nm}$, and $I$ ranges from $\sim 770$ to $1000 \mathrm{~nm}$. The transmittance and response in the UV and IR parts of these bands is further affected by the camera quantum efficiency. On average, the wavelength bands are approximately equivalent to the VRI Johnson-Cousins photometric system. The wavelength range covered is largest for the $V$-equivalent band, thereby providing the highest signalto-noise ratio $(\mathrm{S} / \mathrm{N})$ of the three cameras.

Data from RINGO3 were automatically stacked to produce frames with 10 and $60 \mathrm{~s}$ exposures. Frames were cleaned using the Singular Spectrum Analysis decomposition procedures (Golyandina et al. 2015), to remove vignetting and fringing. All together, the LT data set originally consisted of 65 frames in each of the three color bands. To enhance $\mathrm{S} / \mathrm{N}$ at later times when the afterglow brightness was below $\sim 18 \mathrm{mag}$, frames with $60 \mathrm{~s}$ exposure were co-added from $\sim 10^{3} \mathrm{~s}$ onward.

Because no standard stars' fields were available for the night of the GRB observations, photometric calibration was performed relative to the magnitudes of five non-saturated USNO-B1.0 stars in the field, using standard aperture photometry procedures. USNO I cataloged magnitudes were used to calibrate the $I$-equivalent RINGO3 band, $R 2$ magnitudes to calibrate the $R$-equivalent RINGO3 band, and approximated $V \sim 0.444 \times B 1+0.556 \times R 2$ magnitudes to calibrate the $V$-equivalent RINGO3 band. ${ }^{16}$ While USNO-B1.0 magnitudes provide relatively poor absolute photometric accuracy $( \pm 0.3 \mathrm{mag})$, the photometric stability throughout the observations was very good as shown by the variability in zero points being $<0.1 \mathrm{mag}$ in each RINGO3 band.

\subsection{BOOTES, IAC, OSN, STELLA, and GTC}

The afterglow of GRB 140430A was observed by the following facilities.

The $0.6 \mathrm{~m}$ TELMA robotic telescope at the BOOTES-2 astronomical station at IHSM/UM-CSIC La Mayora, Spain (Castro-Tirado et al. 2012) started observing the afterglow with the COLORES imaging spectrograph at $30 \mathrm{~s}$ after the BAT trigger with $r^{\prime}$ - and $i^{\prime}$-band filters. The detected peak magnitude was $r^{\prime} \sim 16.1 \mathrm{mag}$. Due to poor observing conditions, the

\footnotetext{
15 http://telescope.livjm.ac.uk/TelInst/Inst/RINGO3/

16 The $V$-band estimate using USNO $B$ and $R$ magnitudes is very crude; see www.aerith.net/astro/color_conversion/JG/USNO-B1.0.html.
}

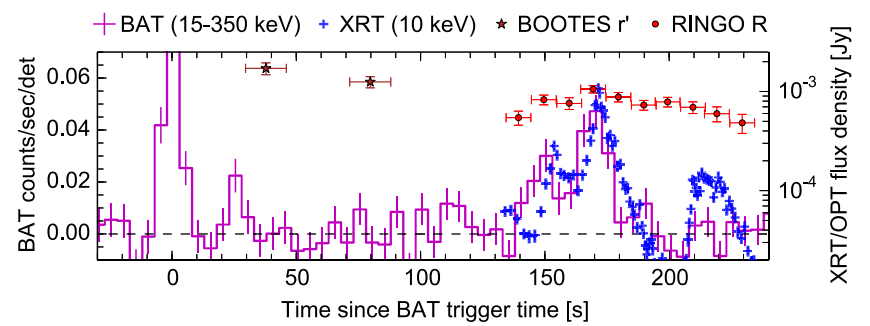

Figure 2. GRB 140430A early light curve in gamma-ray (BAT), X-ray (XRT), and optical bands, including 2 BOOTES epochs.

observations resulted in poor image quality. Nevertheless, we could extract the $r^{\prime}$-band magnitude for two early epochs, which are represented in Figure 2. The first epoch is marginally contemporaneous with the second gamma-ray peak in BAT LC. Due to the significantly poorer temporal sampling of BOOTES data compared to the later optical data, we did not include the BOOTES points in the subsequent detailed LC analysis.

IAC-80 $0.82 \mathrm{~m}$ telescope (Observatorio del Teide) observed the afterglow between 25.0 and 40.9 minutes, providing a $3 \times 300 \mathrm{~s}$ sequence of observations in $B V R$-band filters (Gorosabel et al. 2014).

OSN $1.5 \mathrm{~m}$ telescope (Observatorio de Sierra Nevada) observed the afterglow between 6.7 and 78 minutes, providing 280 frames all together in $I$ band, while gradually extending the exposure time from 5 to $60 \mathrm{~s}$. To enhance signal-to-noise at early times, we coadded the frames to similar binning as the LT frames, while at late times we used $240 \mathrm{~s}$ binning.

STELLA-I $1.2 \mathrm{~m}$ robotic telescope (Observatorio del Teide) observed the afterglow between 2.9 and 32.5 minutes in the $r^{\prime}$ band filter, providing 52 frames with $20 \mathrm{~s}$ exposure times. To enhance the signal-to-noise, we coadded the frames to $60 \mathrm{~s}$ binning at early times and to $120 \mathrm{~s}$ binning at late times.

The $10.4 \mathrm{~m}$ Gran Telescopio Canarias (GTC) telescope observed the afterglow at $\sim 3.5 \times 10^{3} \mathrm{~s}$, obtaining an optical spectra with the OSIRIS imaging spectrograph (Cepa et al. 2000). Data was reduced and calibrated the usual way using IRAF and custom tools coded up in python. We clearly detect several absorption lines, from which a redshift $z=1.600 \pm 0.001$ is derived, consistent with Krühler et al. (2014).

Frames from IAC, OSN, and STELLA telescopes have been calibrated against the same USNO-B1.0 stars and in the same manner as the LT frames. The resulting LCs are plotted in Figure 3. Comparison between data points from the RINGO3 instrument (using dichroics) and data points from other telescopes (using standard filters) confirms that RINGO3 bands are well approximated by the VRI-equivalent photometric system. All calibrated magnitudes were later corrected for the Galactic extinction of $E_{B-V}=0.12 \mathrm{mag}$ in the direction of the burst (Schlafly \& Finkbeiner 2011), and converted to flux densities using Fukugita et al. (1996). The complete photometry is available in Table 4.

\section{ANALYSIS}

\subsection{X-Ray Data}

The X-ray LC shows strong variability and rapid flaring activity at early time. Flaring of the soft gamma-ray emission is also seen at this time, up to $\sim 200 \mathrm{~s}$ post burst, simultaneous with the X-ray flares and showing similar temporal structure in 


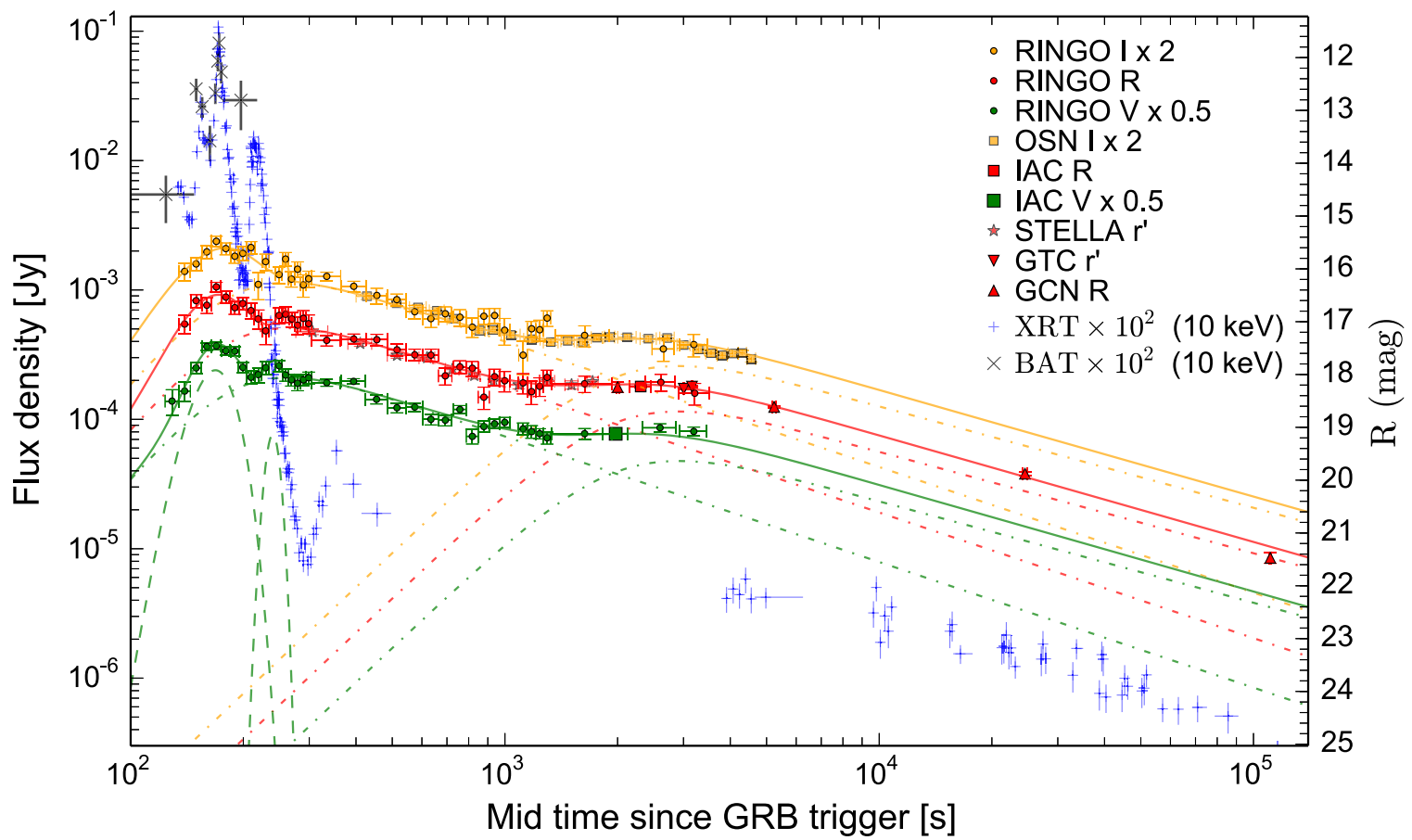

Figure 3. GRB 140430A multi-wavelength light curves in gamma-ray, X-ray, and optical bands. Optical data are best modeled (solid lines) using the sum of 2 Beuermann (dash-dotted lines) profiles to describe broad underlying components and 2 Gaussian (dashed lines) profiles to describe early time flares (Section 3.2).

the LC (Figures 1 and 3). At the end of the steep X-ray decay phase at $\sim 500 \mathrm{~s}$, the X-ray data gap occurs due to Earth occultation and lasts until $4000 \mathrm{~s}$. During this time, the X-ray LC indicates the likely presence of the canonical X-ray afterglow plateau phase (Nousek et al. 2006). From $4000 \mathrm{~s}$ to $\sim 1.3 \times 10^{5} \mathrm{~s}$, the decay is best described by a broken power law with a poorly constraint break time $t_{\mathrm{b}} \sim 9700 \mathrm{~s}$, and with decay indices $\alpha_{1}=0.51 \pm 0.32$ pre-break and $\alpha_{2}=0.85 \pm 0.06$ post-break $\left(\chi_{\text {red }}^{2}=0.99\right)$. The X-ray upper limit obtained at $4.6 \times 10^{5} \mathrm{~s}$ is not consistent with a simple extrapolation of the late time decay, indicating the possible occurrence of a jet break after $10^{5} \mathrm{~s}$.

The time-averaged spectrum formed from the early time data (Windowed Timing-WT mode, see Figure 1) can be well fitted with the absorbed power-law. Fixing the Galactic absorption column to $N_{\mathrm{HI}}^{\mathrm{Gal}}=2.13 \times 10^{21} \mathrm{~cm}^{-2}$ (Willingale et al. 2013), the resulting intrinsic absorption at $z=1.6$ is $N_{\mathrm{HI}}^{\text {Host }}=(3.4 \pm 1.4) \times 10^{21} \mathrm{~cm}^{-2}$, and the power-law photon index of the spectrum is $\Gamma=\beta+1=2.10 \pm 0.04$ (Evans et al. 2009). All values are consistent with the late time data (Photon Counting-PC mode, see Figure 1).

To discuss the early time X-ray emission properties, we modeled the early X-ray LC with a combination of an underlying power-law decay and three superimposed bumps described by the Norris profile (Norris et al. 2005):

$$
\begin{aligned}
F_{\mathrm{X}}(t)= & F_{0}\left(\frac{t}{t_{0}}\right)^{-\alpha_{\text {decay }}} \\
& +\sum_{i=1}^{3} F_{i} \exp \left(2 \sqrt{\frac{\tau_{1, i}}{\tau_{2, i}}}\right) \exp \left(-\frac{\tau_{1, i}}{\tilde{t}}-\frac{\tilde{t}}{\tau_{2, i}}\right),
\end{aligned}
$$

where $F_{0}$ is the power-law normalization factor and $\alpha_{\mathrm{dec}}$ is the overall power-law decay index, $F_{i}$ is a normalization constant of superimposed bumps, $\tau_{1, i}$ and $\tau_{2, i}$ are factors determining the shape of each bump, and $\tilde{t}$ is the time measured from the start of each bump as determined from LC. From parameters of Norris profiles for each bump, it is possible to obtain peak times as $t_{\text {peak }, i}=\sqrt{\tau_{1, i} \tau_{2, i}}$ and peak durations as $\Delta t_{i}=\tau_{2, i} \sqrt{1+4 \sqrt{\tau_{1, i} / \tau_{2, i}}}$ (Norris et al. 2005). The results of fitting this model (Equation (1)) to early X-ray LC are presented in Table 1 . We note that the second X-ray bump is poorly fitted with the Norris profile because the height of the peak is significantly above the fitted function (see Figure 1), but the obtained peak time and width are nevertheless reasonable.

Short variability timescales $(\Delta t / t)$ and large amplitude variability $(\Delta F / F)$ of early time $\mathrm{X}$-ray flares, as presented in Table 1 , are commonly observed in early X-ray LCs for both long and short duration GRBs (Chincarini et al. 2010; Margutti et al. 2010, 2011; Bernardini et al. 2011).

\subsection{Optical $L C$}

Figure 3 shows the calibrated optical LC of GRB 140430A, which is complex and could not be described by a simple power-law behavior. Overall, the LC is qualitatively described by at least two long-lasting emission episodes joined by a plateau phase at $\sim 2000 \mathrm{~s}$. The excellent temporal sampling at early time, however, reveals additional components.

We fitted optical LCs with phenomenological models as typically used in the literature for optical afterglows. Possible theoretical models will be discussed in Section 4. We used a combination of two Beuermann profiles $(B)$, i.e., smoothly connected broken power-laws (Beuermann et al. 1999; Guidorzi et al. 2015a), for broad components, and (following Krühler et al. 2009) two Gaussian profiles $(G)$ corresponding to 
Table 1

Early X-Ray LC Best-fit (Power-law +3 Norris Peaks) Parameters

\begin{tabular}{cccccc}
\hline \hline Peak & $\begin{array}{c}\text { Interval } \\
(\mathrm{s})\end{array}$ & $\begin{array}{c}\text { Peak Time } \\
t_{\text {peak }}(\mathrm{s})\end{array}$ & $\begin{array}{c}\text { Duration } \\
\Delta t(\mathrm{~s})\end{array}$ & $\Delta t / t$ & $\Delta F / F$ \\
\hline 1 & $144-160$ & $154.6 \pm 1.7$ & $18.7 \pm 4.9$ & 0.12 & 1.77 \\
2 & $160-200$ & $173.2 \pm 0.8$ & $22.0 \pm 1.1$ & 0.13 & $6.47^{*}$ \\
3 & $200-496$ & $218.5 \pm 0.7$ & $37.3 \pm 1.0$ & 0.17 & 11.42 \\
\hline
\end{tabular}

Note. Peak times, durations, and flux ratios of three X-ray flares obtained from the early-time fit (see Section 3.1). The underlying power-law component has a decay index of $\alpha_{\text {decay }}=4.3 \pm 0.1$. The $\chi_{\text {red }}^{2}$ of the fit is $\chi_{\text {red }}^{2}=1.19$ with 146 dof. * Because the fit underestimates the flux of the second peak (see the text), $\Delta F / F$ for the second peak could be larger by a factor of $\sim 2$.

early time flares:

$$
\begin{aligned}
F(t)= & \sum_{i=1}^{2} B_{i} \times \frac{1+\alpha_{\mathrm{R}, \mathrm{i}} / \alpha_{\mathrm{D}, \mathrm{i}}}{\left(t / t_{\mathrm{P}, \mathrm{i}}\right)^{-\alpha_{\mathrm{R}, \mathrm{i}}+\left(\alpha_{\mathrm{R}, \mathrm{i}} / \alpha_{\mathrm{D}, \mathrm{i}}\right)\left(t / t_{\mathrm{P}, \mathrm{i}}\right)^{\alpha_{\mathrm{D}, \mathrm{i}}}}} \\
& +\sum_{j=1}^{2} G_{j} \times \exp \left(\frac{-\left(t-t_{\mathrm{P}, \mathrm{j}}\right)^{2}}{2 \sigma_{j}^{2}}\right),
\end{aligned}
$$

where $B_{i}$ and $G_{j}$ are normalization constants, $t_{\mathrm{P}}$ are peak times, $\alpha_{\mathrm{R}}$ are rise indices, and $\alpha_{\mathrm{D}}$ are decay indices. Parameters $\sigma_{j}$ from Gaussian profiles can be connected with the overall duration of the bump as $\Delta t \approx 2 \times \mathrm{FWHM} \approx 2 \times 2 \sqrt{2 \ln 2} \sigma$. Note that we fixed the smoothing parameter from a more general Beuermann equation to $s=1$, due to not very well constrained peak shapes of broad Beuermann components (using $s=0.5$ and $s=2$ does not change results). We also note that the Gaussian description of the temporal profile is not physically motivated, but provides reasonable values of peak time and width.

We fitted all three wavelength bands simultaneously with different normalization factors for each color. For Beuermann profiles, we assumed common $t_{\mathrm{P}, \mathrm{i}}, \alpha_{\mathrm{R}, \mathrm{i}}$, and $\alpha_{\mathrm{D}, \mathrm{i}}$, while, for Gaussian profiles, we assumed different $t_{\mathrm{P}, \mathrm{j}}$ and $\sigma_{j}$ for each color. We used the Levenberg-Marquardt algorithm, assuming that photometric uncertainties are normally distributed. The fitting method provides the best-fit values and $1 \sigma$ uncertainties of free parameters (Table 2). The resulting $\chi^{2}=168$ with degrees of freedom (dof) $=125$ has low $P$-value of $P=0.006$, indicating that the model does not describe our complex data set well. Nevertheless, when testing different models by adding or removing Beuermann and/or Gaussian components, the fit did not improve and the residuals increased.

To obtain additional information from the fitted model, especially the power-law rise and decay indices, both data and best-fit models are represented in differential plots in $\log -\log$ scale (Figure 4). Although data show large scatter and relatively large uncertainties, which results in large scatter in the differential plot, the best-fit model shows that during the first optical flare the initial rising part power-law index is between $\alpha_{\text {rise }} \sim 4$ and $\alpha_{\text {rise }} \sim 6$ (depending on wavelength), followed by a decay with a power-law index between $\alpha_{\text {decay }} \sim 2$ and $\alpha_{\text {decay }} \sim 4$. During the second optical flare, both rising and decaying indices are around $\sim 5$ (the $I$-band fit is not very constraining for the second flare). The LC then shows more smooth behavior with a decaying power-law index $\alpha_{\text {decay }} \sim 1$ from $\sim 300 \mathrm{~s}$ to $\sim 10^{3} \mathrm{~s}$. We note that at that time, the afterglow has dimmed for more than $1.5 \mathrm{mag}$ and
Table 2

Optical LC Best-fit (2 Beuermann +2 Gaussian) Parameters

\begin{tabular}{|c|c|c|c|c|}
\hline \multirow[b]{2}{*}{ Peak } & \multicolumn{3}{|c|}{ Beuermann Peaks (Underlying Components): } & \multirow[b]{2}{*}{$F_{\mathrm{p}}(\mathrm{mJy}$} \\
\hline & Peak Time (s) & $\alpha_{\text {rise }}$ & $\alpha_{\text {decay }}$ & \\
\hline $1, V$ & $\sim 260$ & $3.37 \pm 1.65$ & $0.97 \pm 0.12$ & 0.420 \\
\hline $1, R$ & & & & 0.507 \\
\hline $1, I$ & & & & 0.569 \\
\hline $2, V$ & $\sim 2900$ & $2.73 \pm 0.44$ & $0.79 \pm 0.03$ & 0.095 \\
\hline $2, R$ & & & & 0.115 \\
\hline $2, I$ & & & & 0.129 \\
\hline \multicolumn{5}{|c|}{ Gaussian Peaks (Early Time Flares): } \\
\hline Peak & Peak Time (s) & Duration (s) & $\Delta t / t$ & $\Delta F / F$ \\
\hline $1, V$ & $\begin{array}{c}168.4 \pm 2.7 \\
\alpha_{\text {rise }}=6.2\end{array}$ & $\begin{array}{l}95.9 \pm 21.1 \\
\alpha_{\text {decay }}=3.8\end{array}$ & $0.57 \pm 0.13$ & 1.72 \\
\hline $1, R$ & $\begin{array}{c}166.5 \pm 4.5 \\
\alpha_{\text {rise }}=5.0\end{array}$ & $\begin{array}{c}133.4 \pm 25.8 \\
\alpha_{\text {decay }}=2.7\end{array}$ & $0.80 \pm 0.16$ & 1.76 \\
\hline $1, I$ & $\begin{array}{c}170.6 \pm 6.7 \\
\alpha_{\text {rise }}=4.2\end{array}$ & $\begin{array}{c}175.9 \pm 31.1 \\
\alpha_{\text {decay }}=2.2\end{array}$ & $1.03 \pm 0.19$ & 1.68 \\
\hline $2, V$ & $\begin{array}{c}239.7 \pm 3.8 \\
\alpha_{\text {rise }}=4.4\end{array}$ & $\begin{array}{l}46.4 \pm 23.2 \\
\alpha_{\text {decay }}=4.5\end{array}$ & $0.19 \pm 0.10$ & 0.37 \\
\hline $2, R$ & $\begin{array}{c}257.8 \pm 5.5 \\
\alpha_{\text {rise }}=4.2\end{array}$ & $\begin{array}{l}47.0 \pm 26.9 \\
\alpha_{\text {decay }}=5.0\end{array}$ & $0.18 \pm 0.10$ & 0.37 \\
\hline $2, I$ & $\begin{array}{c}259.3 \pm 7.5 \\
\alpha_{\text {rise }}=8.7\end{array}$ & $\begin{array}{c}20.9 \pm 17.5 \\
\alpha_{\text {decay }}=11.0\end{array}$ & $0.08 \pm 0.07$ & 0.43 \\
\hline
\end{tabular}
Beuermann Peaks (Underlying Components):

Note. The fit was performed simultaneously in $V, R$, and $I$ bands, with peak times, $\alpha_{\text {rise }}, \alpha_{\text {decay }}$ of the Beuermann profiles taken as common parameters among all three bands. Parameters for Gaussian profiles (peak time and width) were taken differently for each band. $F_{\mathrm{p}}$ is the flux density at the time of the peak for each of the two Beuermann profiles, and although the peak flux density is different for each band, we assumed a common flux density ratio between the first and the second Beuermann peak for all bands (the ratio obtained from the fit is $0.23 \pm 0.03) . \Delta t / t$ is calculated as duration divided by peak time. $\Delta F / F$ is calculated as the ratio between difference of flux density at the time of the Gaussian peak and flux density of the underlying Beuermann peak, divided by flux density of the underlying Beuermann peak. Based on Figure 4 , estimates of the maximum power-law rise $\left(\alpha_{\text {rise }}\right)$ and decay $\left(\alpha_{\text {decay }}\right)$ indices for Gaussian peaks are also given for each optical band.

photometric uncertainties, especially for RINGO3 frames due to low sensitivity, become larger, but OSN I-band and STELLA $r^{\prime}$-band points, which are available at that time, are of much better quality and thus more constraining. The differential plot between $\sim 1000$ and $\sim 3000$ s thus shows a smooth transition from decay to a plateau phase, and then back to decay phase with a slightly shallower decay index $\alpha_{\text {decay }} \sim 0.8$.

\subsection{Broadband Spectral Energy Distribution (SED)}

At early times, due to simultaneous sampling of the LC in three visible wavelength bands and in the X-ray band, we could study the temporal evolution of SED. The spectral index of the $\mathrm{X}$-ray emission $\left(\beta_{\mathrm{X}}\right)$, obtained from the Swift Burst Analyser (Evans et al. 2010), can be compared to the power-law slope of the optical SED $\left(\beta_{\mathrm{OPT}}\right)$, obtained from fitting a power law to optical data points, or to the broadband optical to X-ray extrapolated spectral index $\left(\beta_{\mathrm{R}-\mathrm{X}}\right)$, obtained from fitting a power-law to optical $R$-band and contemporaneous $\mathrm{X}$-ray $(10 \mathrm{keV})$ flux densities. We neglected the contribution of host galaxy extinction in optical bands, due to its low upper limit 


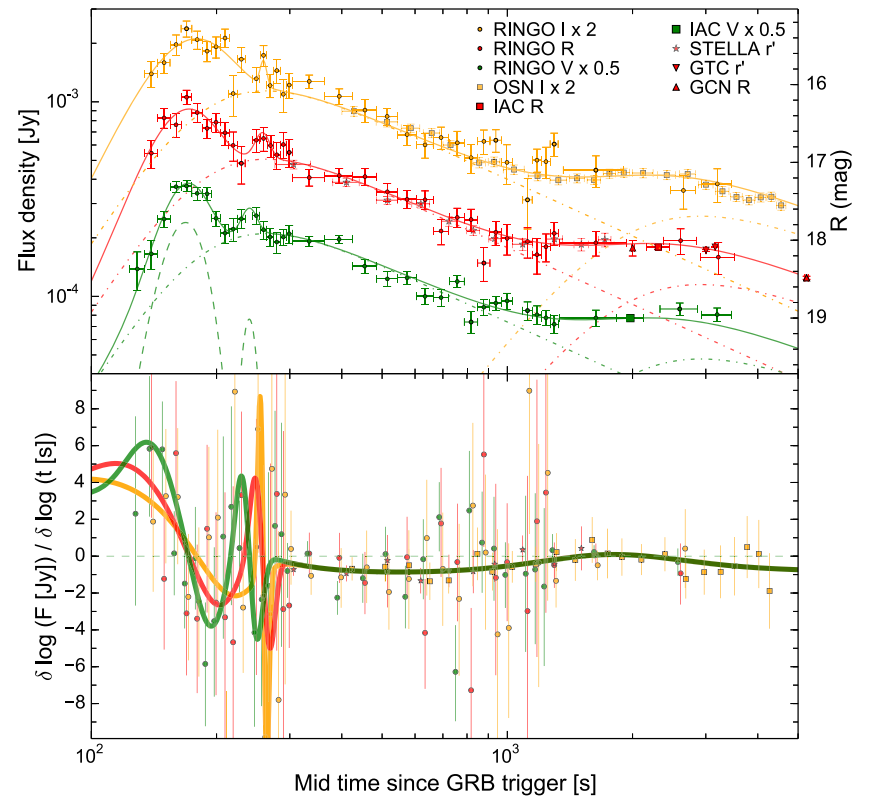

Figure 4. GRB 140430A optical light curves in three bands, together with the derivative plot in the $\log -\log$ scale. The derivative plot clearly shows deviations from a simple power-law behavior at early times, which corresponds to early time optical flares. For results obtained from this plot, see Section 3.2.

(see the end of this section and Figure 6). SED evolution is presented in Figure 5.

We see from Figure 5 (bottom panel) that at early times the $\mathrm{X}$-ray spectral index is highly variable and that it tracks the $\mathrm{X}$-ray LC throughout the flares. The hard-to-soft spectral evolution is observed. At the end of the last prominent X-ray flare at $\sim 240 \mathrm{~s}$ the X-ray emission becomes extremely soft $\left(\beta_{\mathrm{X}} \sim 2.5\right)$ during the steep decay phase, but then it hardens at later times, when flaring is no longer present, and stays around the value of $\beta_{\mathrm{X}}=1.10 \pm 0.04$, as obtained from the late time XRT spectral fit (Evans et al. 2009) and consistent with our late time SED fit (Figure 6).

The temporal evolution of optical to X-ray extrapolated index $\left(\beta_{\mathrm{R}-\mathrm{X}}\right)$ and optical spectral index $\left(\beta_{\mathrm{OPT}}\right)$ helps us understand if emission powering early time $\mathrm{X}$-ray flares also manifests itself in the optical domain. The first thing that we notice is that at early times the variability of the spectral index in broadband optical to X-ray SED is not as prominent as in the $\mathrm{X}$-ray alone. The emission tends to be harder at the beginning, changing to softer at later times (after $\sim 400 \mathrm{~s}$ ), when it is consistent with $\beta_{\mathrm{X}}$.

The temporal behavior of the optical spectral index $\left(\beta_{\mathrm{OPT}}\right)$ suggests some degree of variability at early times but it is difficult to quantify this given the uncertainties. The statistical significance of variations in $\beta_{\mathrm{OPT}}$ is low, as the constant fit to $\beta_{\text {OPT }}$ over the time interval $140-450$ s gives an acceptable fit of average spectral index $\beta_{\mathrm{OPT}}=0.97 \pm 0.08$, with reduced $\chi^{2}$ of 1.4 and $P$-value of 0.12 . After $300 \mathrm{~s}$ the spectral index implied by the best fitting model converges to $\beta_{\mathrm{OPT}}=$ $0.65 \pm 0.06$, while the variations implied by the RINGO3 data at later times become less constraining due to large uncertainties.

We built late time broadband SED at $\sim 3200 \mathrm{~s}$, when photometric data in four optical bands are available. We took X-ray data from the XRT spectra repository (Evans et al. 2009) in the time interval $3-30 \mathrm{ks}$, in which there is no spectral

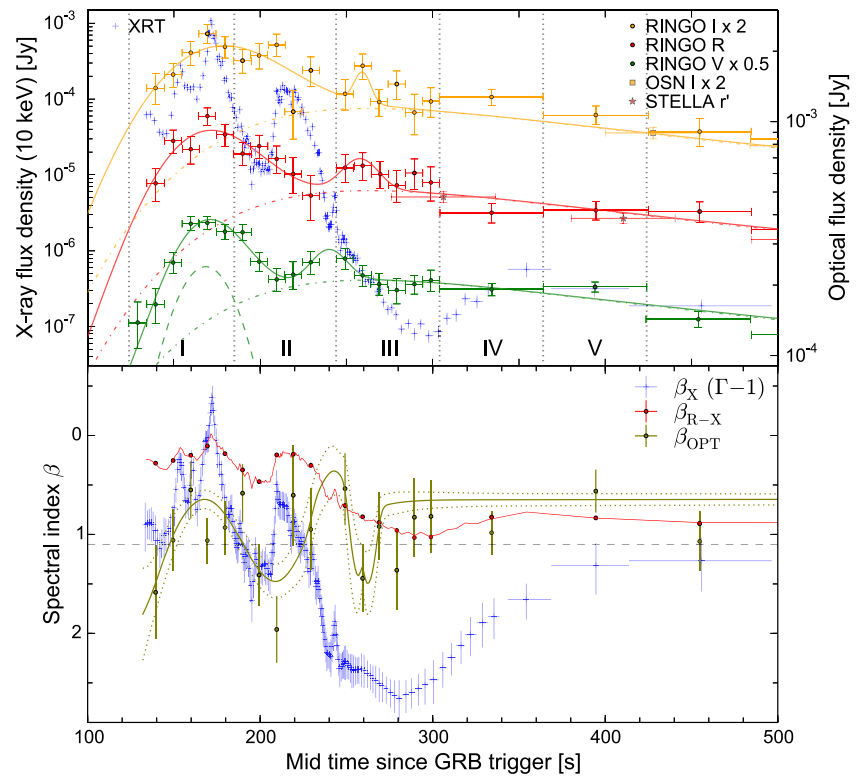

Figure 5. GRB 140430A early time X-ray and optical light curves in linear temporal scale (the optical flux density scale has been stretched and is represented on the right axis), together with the spectral power-law index of the $\mathrm{X}$-ray emission $\left(\beta_{\mathrm{X}}\right)$, optical emission $\left(\beta_{\mathrm{OPT}}\right)$, and optical to X-ray extrapolated index $\left(\beta_{\mathrm{R}-\mathrm{X}}\right)$. The red solid line connecting $\beta_{\mathrm{R}-\mathrm{X}}$ points is obtained by fitting a power-law SED to best-fit $R$-band model and X-ray points. The olive solid line connecting $\beta_{\text {OPT }}$ points is obtained by fitting a power-law SED to best-fit optical models, and the corresponding dotted olive lines represent the $1 \sigma$ confidence interval of the fit. The dashed horizontal line corresponds to late time $\beta_{\mathrm{X}} \approx 1.1$. Vertical dotted lines in the top panel show five time epochs where polarimetry has been performed (see Section 3.4 and Table 3). For a discussion on SED analysis, see Section 3.3.

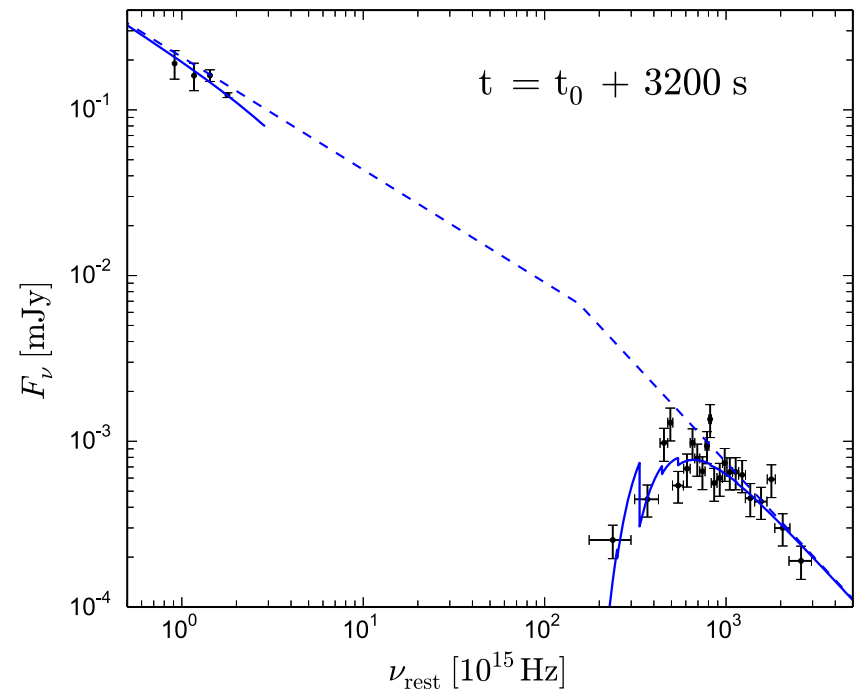

Figure 6. GRB 140430A late time spectral energy distribution, with best-fit results (see the text) plotted with blue lines. The solid line also takes into account optical extinction and soft-X-ray absorption.

evolution. The mean time was computed as $\sum_{i}\left(t_{i} \Delta t_{i}\right) / \sum_{i} \Delta t_{i}$, where $t_{i}$ is the mid-time of individual exposure and $\Delta t_{i}$ is the exposure time. By knowing the temporal power-law slope, we normalized obtained flux densities to the epoch of optical observations. Using XSPECV12.8, we fitted the Milky Way, Large Magellanic Cloud, and Small Magellanic Cloud (SMC) average extinction curves (Pei 1992) to our data set, combined with a single or a broken power-law slope. The best fit was 

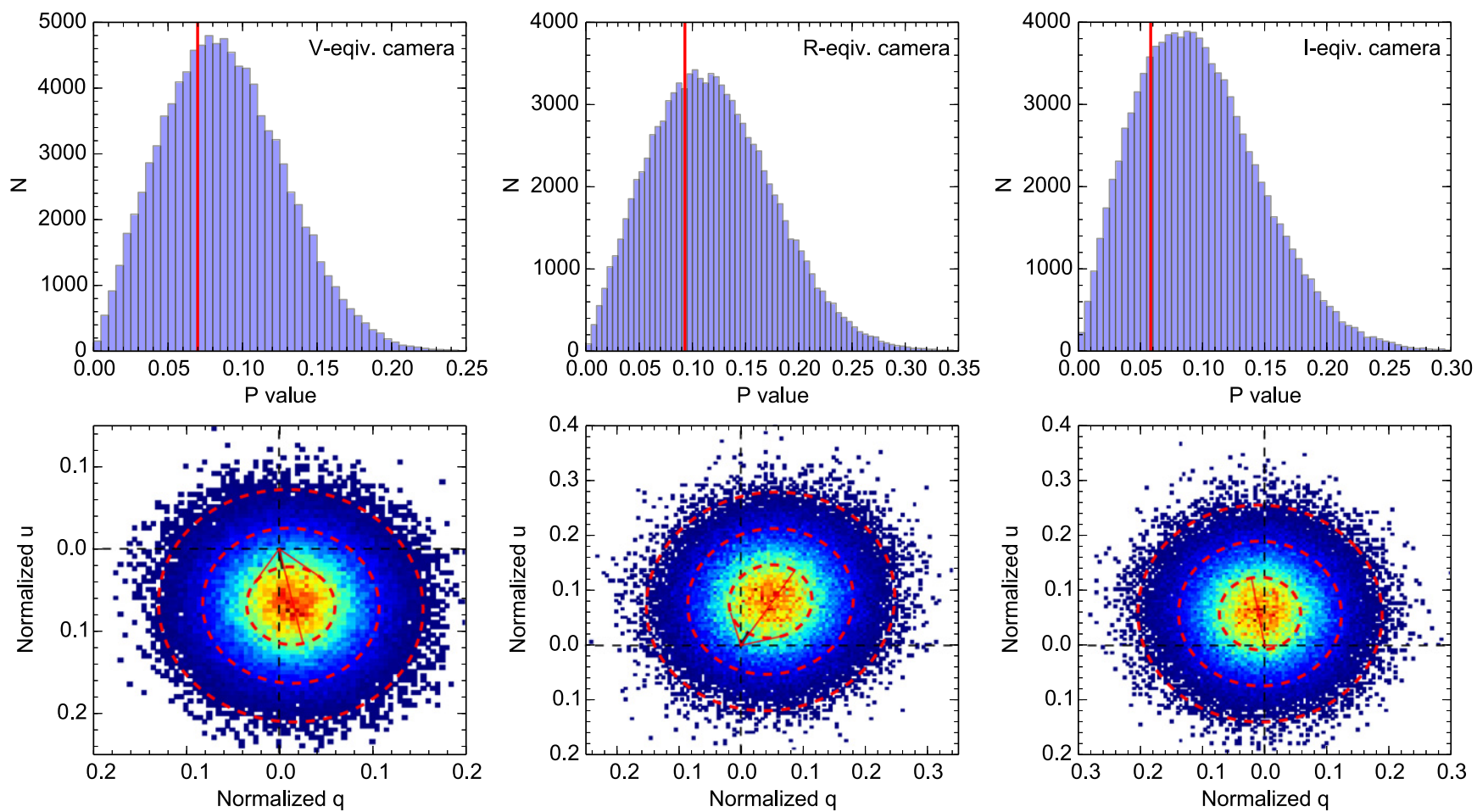

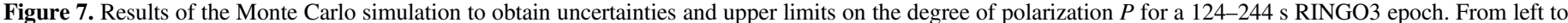

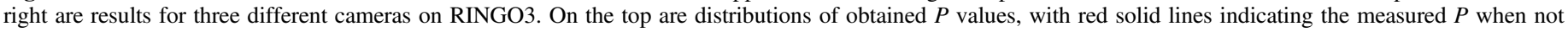

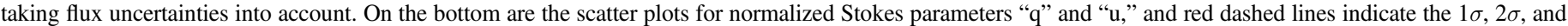
$3 \sigma$ contours (from center outward), obtained by fitting a $2 \mathrm{D}$ normal distribution.

obtained using a broken power-law and SMC extinction profile, which is most common for GRB SEDs (e.g., Japelj et al. 2015 and references therein). By fixing the difference of power-law slopes to $\Delta \beta=0.5$ and fixing $N_{\mathrm{H}, \mathrm{X}}^{\mathrm{Gal}}=2.13 \times 10^{21} \mathrm{~cm}^{-2}$ (Willingale et al. 2013), we obtained the following best-fit parameters: $\beta_{1}=0.68_{-0.14}^{+0.13}, \quad \beta_{2}=1.18, \quad \nu_{\text {break }}=160_{-140}^{+800} \times$ $10^{15} \mathrm{~Hz}, \quad N_{\mathrm{H}, \mathrm{X}}<8 \times 10^{21} \mathrm{~cm}^{-2}, \quad$ and $A_{\mathrm{V}}<0.14$, with $\chi^{2} /$ d. o. $\mathrm{f}=24.8 / 21$. The resulting late time broadband SED is presented in Figure 6.

\subsection{Polarization}

At the time of our measurements (2014 April 30th), the RINGO3 polarimeter was in the process of commissioning. The data were taken when the full sensitivity of the instrument was not reached, resulting in low $\mathrm{S} / \mathrm{N}$ obtained from our measurement. Consequently, only the upper limits could be obtained for polarization degree $P$.

The polarimetry was done using standard RINGO procedures (D. M. Arnold et al. 2015, in preparation), similarly as for GRB 120308A (Mundell et al. 2013), by first correcting the obtained Stokes parameters for instrumental-induced polarization and then by correcting the obtained polarization degree for instrumental depolarization. Corrections are obtained from the analysis of a full set of polarimetric standard stars.

To correctly obtain the uncertainties $( \pm 1 \sigma)$ on the degree of polarization $P$, we performed a Monte Carlo simulation taking into account normal distributed photometric uncertainties in each of the eight polaroid orientations. By generating 100,000 simulated flux values, we calculated the corresponding normalized Stokes parameters "q" and "u" following Clarke \& Neumayer (2002). By fitting a 2D normal distribution to "qu" plane, we obtained the mean "q" and " $u$ " values and $\sigma$ contours, which were then used to determine the upper limits on $P$. Figure 7 shows both the distribution of obtained $P$ values from these simulations and the "qu" scatter plots, for the RINGO3 epoch from 124 to $244 \mathrm{~s}$. The final values on polarization degree for GRB 140430A at various RIGNO3 epochs from 124 to $424 \mathrm{~s}$ are summarized in Table 3 .

\section{DISCUSSION}

\subsection{Early Flares}

We discuss the behavior of the LCs at early time in more detail and show that the initial optical flares appear to be more consistent with a prompt rather than afterglow origin.

\subsubsection{Prompt Origin}

The early optical LC is dominated by highly variable components, which are not easily explained in the pure context of the standard external-shock afterglow scenario (either from forward- or reverse-shock), where smooth behavior is expected with rise index $\alpha_{\text {rise }}<5$ and decay index $\alpha_{\text {decay }}<2-3$ (e.g., Kobayashi 2000; Kobayashi \& Zhang 2003; Zhang et al. 2003). Although temporal rise and decay indices for the first optical flare $\left(\alpha_{\text {rise }} \sim 4.2-6.2\right.$ and $\alpha_{\text {decay }} \sim 2.2-3.8$, depending on the wavelength band) could be marginally consistent with those predicted by afterglow models, the indices are much steeper for the second optical flare $\left(\alpha_{\text {rise }} \sim 4.2-8.7\right.$ and $\alpha_{\text {decay }} \sim$ 4.5-11.8, see Section 3.2). The underlying broad Beuermann component points toward the origin of the emission from external shock afterglow, but the superimposed optical components, which appear during the on-going high-energy gamma-ray and X-ray flares, point at least partially toward an internal shock origin. 
Table 3

GRB 140430A Optical Polarization Results

\begin{tabular}{lccc}
\hline \hline Interval (s) & $V$-eq. Band & $R$-eq. Band & $I$-eq. Band \\
\hline $124-185$ & $<19 \%$ & $<19 \%$ & $<14 \%$ \\
$185-244$ & $<20 \%$ & $<16 \%$ & $<16 \%$ \\
$244-304$ & $<22 \%$ & $<12 \% *$ & $<22 \%$ \\
$304-364$ & $<23 \%$ & $<20 \%$ & $<17 \%$ \\
$364-424$ & $<17 \%$ & $<16 \%$ & $<10 \%$ \\
$124-244^{*}$ & $<12 \%$ & $3 \sigma<30 \%$ & $3 \sigma<26 \%$ \\
& $3 \sigma<22 \%$ & & $<12 \%$ \\
\hline
\end{tabular}

Note. $1 \sigma$ (unless stated otherwise) upper limits on early time optical polarization degree $P$ from three RINGO3 cameras, in various time intervals.

* indicates that data have been coadded in two time intervals, to obtain a better $\mathrm{S} / \mathrm{N}$.

It has been suggested by studying X-ray flares that those can originate due to dissipation within the internal shock region (e.g., Burrows et al. 2005; Zhang et al. 2006; Chincarini et al. 2007; Troja et al. 2015), similarly to gamma-ray emission (Rees \& Mészáros 1992). A recent statistical study of the waiting time distribution between gamma-ray pulses and X-ray flares showed that both phenomena are linked and likely produced by the same mechanism (Guidorzi et al. 2015b). Especially short variability timescales $(\Delta t / t)$ and large amplitude variability $(\Delta F / F)$ disfavor the origin of X-ray flares from the afterglow region due to density fluctuations, refreshed shocks, or patchy shells (Ioka et al. 2005). Values of $\Delta t / t$ and $\Delta F / F$ for early X-ray flares (Table 1) fall outside of kinematically allowed regions for afterglow variability (Ioka et al. 2005).

The study by Kopač et al. (2013) showed that optical emission at early times, especially when showing sharp and steep peaks in LCs, can also originate from dissipation within internal shocks. Based on a simple two-shell internal shock collision model, the distribution of the flux ratio between high-energy and optical emission can span from $\left(\nu F_{\nu}\right)^{\gamma} /\left(\nu F_{\nu}\right)^{\mathrm{OPT}} \gtrsim 1$ to $\left(\nu F_{\nu}\right)^{\gamma} /\left(\nu F_{\nu}\right)^{\mathrm{OPT}} \lesssim 10^{5}$, depending on various parameters like the bulk Lorentz factor of the ejected shell, energy density of electrons, energy density of magnetic fields, etc. The temporal delay of peaks at different energies can be due to different radii of shell collisions, depending on the initial separation between shells and the distribution of Lorentz factors. The flux ratio between $\mathrm{X}$-ray and $R$-band for GRB $140430 \mathrm{~A}$ is $\left(\nu F_{\nu}\right)^{\mathrm{X}} /\left(\nu F_{\nu}\right)^{\mathrm{R}}=1.5 \times 10^{4}$ for the first optical flare, and $\left(\nu F_{\nu}\right)^{\mathrm{X}} /\left(\nu F_{\nu}\right)^{\mathrm{R}}=2 \times 10^{3}$ for the second optical flare, indicating that the amount of energy emitted in optical bands is relatively small. Such flux ratio values are consistent with the values from the sample study of Kopač et al. (2013), and are comparable to, for example, GRB 080928 (Rossi et al. 2011) and GRB 110205A (Gendre et al. 2012; Zheng et al. 2012). Similarly to other GRBs that show prompt optical flares, values of $\Delta t / t$ (Table 2 ) are below 1 , and even below 0.2 for the second optical flare.

Strong spectral evolution is commonly observed in timeresolved spectra of prompt gamma-ray emission (e.g., Lu et al. 2012) and X-ray flares (e.g., Butler \& Kocevski 2007; Zhang et al. 2007). The variability timescale of spectral behavior is short, similar to the corresponding LC behavior, and different from that typically observed in the afterglow regime, where variations are usually smooth and the spectral slope stays constant or changes at breaks according to standard afterglow theory (Sari et al. 1998). The X-ray spectral index $\left(\beta_{\mathrm{X}}\right)$ shows high variability during the prompt phase, and a hard-to-soft spectral evolution that tracks the flares (see Figure 5), pointing toward an internal shock origin. Variability in the optical spectral index $\left(\beta_{\mathrm{OPT}}\right)$ at early times is also suggested by the data but large uncertainties (Figure 5, olive points) prevent confirmation at a statistically significant level (see Section 3.3). In contrast, the spectral index of the broadband optical to X-ray $\operatorname{SED}\left(\beta_{\mathrm{R}-\mathrm{X}}\right)$ changes much more smoothly, with a gradual softening of the emission with time. This is likely due to the fact that early flares, which are much more powerful in the X-ray part of the spectrum, can mask the underlying synchrotron component from the afterglow emission, which is more prominent in the optical regime.

Based on the discussion of a strong optical flare from GRB 080129 (Greiner et al. 2009), likely causes for optical flares at early times could also be residual collisions ( $\mathrm{Li} \&$ Waxman 2008), which predict variability on a timescale of the same order as the delay between gamma-ray and optical emission, or Poynting flux dissipation (Giannios 2006; Lyutikov 2006), which, in the case of GRB 140430A, is unlikely due to the lack of very high polarization.

\subsubsection{Afterglow Origin}

An alternative scenario for the first optical flare is emission from an external shock, possibly reverse-shock emission. Examining the temporal behavior and following Japelj et al. (2014), we neglected the second optical flare, normalized the optical LCs to a common band using normalization parameters from Table 2, and modeled the resultant data set with a set of reverse- plus forward-shock LCs, assuming a thin- or thickshell limit and interstellar medium (ISM) environment of constant density (Kobayashi 2000; Zhang et al. 2003; Japelj et al. 2014; Figure 8). The values emerging from the best model are $t_{\text {peak, } \mathrm{FS}} \approx 444 \mathrm{~s}, \quad t_{\text {peak }, \mathrm{RS}} \approx 162 \mathrm{~s}, \quad R_{\mathrm{B}} \equiv \epsilon_{\mathrm{B}, \mathrm{r}} / \epsilon_{\mathrm{B}, \mathrm{f}} \approx 2.5$, $p \approx 2.3, \epsilon_{\mathrm{e}} \approx 0.2, \Gamma_{0} \sim 40$, however, the modeling cannot sufficiently explain the data set, as indicated by the resultant residuals that suggest the presence of an additional emission component during the first optical peak. Another contradiction comes from the fact that the initial steep rise $\alpha \sim 5$ can only be explained by the thin-shell case, but the fact that the duration of the burst $\left(T_{90} \sim 174 \mathrm{~s}\right)$ is larger than the peak time of the optical emission strongly suggests the thick-shell case (Zhang et al. 2003). Furthermore, the initial rise is too steep to be explained by the reverse-shock from the wind-type environment (Kobayashi \& Zhang 2003).

A complementary test of external shock emission is that of color evolution. In the context of reverse shock emission, no color evolution is expected because the reverse shock peak is typically attributed to the change in dynamics of the ejecta, rather than due to the passage of the spectral break, unless these two events coincide. However, the combination of two peaks as presented in Figure 8 indicates that the forward shock peak should be due to the typical synchrotron passage, and a change in spectral index from $\beta=-1 / 3$ to $\beta=(p-1) / 2$ is expected (Sari et al. 1998). Using the three-band optical data for GRB 140430A, we searched for color evolution around the optical peak times. As can be seen in Figure 5, no such color evolution as predicted by the theory is observed during the apparent forward shock peak. 
Table 4

GRB 140430A: Photometry

\begin{tabular}{|c|c|c|c|c|c|}
\hline$t_{\text {mid }}(\mathrm{s})$ & $\operatorname{Exp}(\mathrm{s})$ & Telescope & Band & Magnitude & $F_{\nu}^{\mathrm{OPT}}(\mathrm{mJy})$ \\
\hline 139.4 & 10 & LT & $I$ & $16.63 \pm 0.17$ & $0.696 \pm 0.106$ \\
\hline 149.6 & 10 & LT & $I$ & $16.48 \pm 0.13$ & $0.795 \pm 0.095$ \\
\hline 159.8 & 10 & $\mathrm{LT}$ & $I$ & $16.25 \pm 0.13$ & $0.985 \pm 0.118$ \\
\hline 169.4 & 10 & $\mathrm{LT}$ & $I$ & $16.04 \pm 0.1$ & $1.188 \pm 0.113$ \\
\hline 179.6 & 10 & LT & $I$ & $16.18 \pm 0.11$ & $1.044 \pm 0.11$ \\
\hline 189.8 & 10 & $\mathrm{LT}$ & $I$ & $16.33 \pm 0.13$ & $0.911 \pm 0.107$ \\
\hline 199.4 & 10 & LT & $I$ & $16.28 \pm 0.14$ & $0.959 \pm 0.119$ \\
\hline 209.6 & 10 & LT & $I$ & $16.16 \pm 0.12$ & $1.064 \pm 0.118$ \\
\hline 219.2 & 10 & $\mathrm{LT}$ & $I$ & $16.9 \pm 0.26$ & $0.551 \pm 0.131$ \\
\hline 229.4 & 10 & LT & $I$ & $16.44 \pm 0.16$ & $0.828 \pm 0.118$ \\
\hline 249.2 & 10 & $\mathrm{LT}$ & $I$ & $16.69 \pm 0.16$ & $0.657 \pm 0.097$ \\
\hline 259.4 & 10 & $\mathrm{LT}$ & $I$ & $16.39 \pm 0.13$ & $0.867 \pm 0.107$ \\
\hline 269 & 10 & LT & $I$ & $16.77 \pm 0.15$ & $0.607 \pm 0.08$ \\
\hline 279.2 & 10 & LT & $I$ & $16.58 \pm 0.15$ & $0.724 \pm 0.1$ \\
\hline 289.4 & 10 & $\mathrm{LT}$ & $I$ & $16.9 \pm 0.22$ & $0.547 \pm 0.107$ \\
\hline 299 & 10 & LT & $I$ & $16.77 \pm 0.16$ & $0.61 \pm 0.089$ \\
\hline 334.1 & 60 & LT & $I$ & $16.71 \pm 0.08$ & $0.637 \pm 0.049$ \\
\hline 394.5 & 61 & $\mathrm{LT}$ & $I$ & $16.91 \pm 0.1$ & $0.534 \pm 0.05$ \\
\hline 454.7 & 60 & $\mathrm{LT}$ & $I$ & $17.09 \pm 0.15$ & $0.453 \pm 0.062$ \\
\hline 514.1 & 60 & LT & $I$ & $17.17 \pm 0.11$ & $0.421 \pm 0.044$ \\
\hline 574.1 & 60 & $\mathrm{LT}$ & $I$ & $17.4 \pm 0.13$ & $0.34 \pm 0.04$ \\
\hline 634.1 & 60 & LT & $I$ & $17.54 \pm 0.16$ & $0.3 \pm 0.044$ \\
\hline 692.9 & 57 & $\mathrm{LT}$ & $I$ & $17.44 \pm 0.14$ & $0.328 \pm 0.043$ \\
\hline 758.1 & 61 & $\mathrm{LT}$ & $I$ & $17.51 \pm 0.16$ & $0.308 \pm 0.044$ \\
\hline 818.3 & 60 & $\mathrm{LT}$ & $I$ & $17.71 \pm 0.18$ & $0.258 \pm 0.043$ \\
\hline 878.3 & 60 & $\mathrm{LT}$ & $I$ & $17.49 \pm 0.16$ & $0.313 \pm 0.045$ \\
\hline 938.3 & 60 & $\mathrm{LT}$ & $I$ & $17.48 \pm 0.15$ & $0.317 \pm 0.042$ \\
\hline 998.1 & 61 & LT & $I$ & $17.78 \pm 0.26$ & $0.244 \pm 0.057$ \\
\hline 1118.3 & 60 & $\mathrm{LT}$ & $I$ & $18.28 \pm 0.33$ & $0.157 \pm 0.046$ \\
\hline 1178.3 & 60 & LT & $I$ & $17.74 \pm 0.15$ & $0.251 \pm 0.035$ \\
\hline 1238.3 & 60 & $\mathrm{LT}$ & $I$ & $17.76 \pm 0.2$ & $0.246 \pm 0.046$ \\
\hline 1296.5 & 57 & $\mathrm{LT}$ & $I$ & $17.53 \pm 0.15$ & $0.303 \pm 0.042$ \\
\hline 1632.5 & 537 & $\mathrm{LT}$ & $I$ & $17.87 \pm 0.2$ & $0.223 \pm 0.041$ \\
\hline 2650.1 & 356 & $\mathrm{LT}$ & $I$ & $18.14 \pm 0.22$ & $0.175 \pm 0.035$ \\
\hline 3196.6 & 537 & $\mathrm{LT}$ & $I$ & $18.05 \pm 0.22$ & $0.189 \pm 0.037$ \\
\hline 427.8 & 55 & OSN & $I$ & $17.1 \pm 0.06$ & $0.447 \pm 0.026$ \\
\hline 513.8 & 60 & OSN & $I$ & $17.23 \pm 0.06$ & $0.395 \pm 0.023$ \\
\hline 585.9 & 60 & OSN & $I$ & $17.31 \pm 0.05$ & $0.366 \pm 0.018$ \\
\hline 658 & 60 & OSN & $I$ & $17.38 \pm 0.06$ & $0.346 \pm 0.019$ \\
\hline 730.3 & 60 & OSN & $I$ & $17.53 \pm 0.06$ & $0.3 \pm 0.016$ \\
\hline 855.9 & 60 & OSN & $I$ & $17.76 \pm 0.07$ & $0.243 \pm 0.015$ \\
\hline 929.7 & 60 & OSN & $I$ & $17.75 \pm 0.06$ & $0.246 \pm 0.014$ \\
\hline 1037.9 & 120 & OSN & $I$ & $17.85 \pm 0.06$ & $0.224 \pm 0.012$ \\
\hline 1182.1 & 120 & OSN & $I$ & $17.93 \pm 0.06$ & $0.208 \pm 0.012$ \\
\hline 1326.4 & 120 & OSN & $I$ & $17.98 \pm 0.06$ & $0.198 \pm 0.011$ \\
\hline 1471.1 & 120 & OSN & $I$ & $17.96 \pm 0.06$ & $0.202 \pm 0.011$ \\
\hline 1615.4 & 120 & OSN & $I$ & $17.98 \pm 0.06$ & $0.198 \pm 0.011$ \\
\hline 1760.1 & 120 & OSN & $I$ & $17.9 \pm 0.06$ & $0.214 \pm 0.011$ \\
\hline 1904.9 & 120 & OSN & $I$ & $17.89 \pm 0.06$ & $0.216 \pm 0.011$ \\
\hline 2121.2 & 240 & OSN & $I$ & $17.89 \pm 0.06$ & $0.215 \pm 0.012$ \\
\hline 2416.9 & 240 & OSN & $I$ & $17.92 \pm 0.06$ & $0.21 \pm 0.011$ \\
\hline 2713.1 & 240 & OSN & $I$ & $17.9 \pm 0.06$ & $0.213 \pm 0.011$ \\
\hline 3002.8 & 240 & OSN & $I$ & $18.04 \pm 0.06$ & $0.188 \pm 0.01$ \\
\hline 3289.9 & 240 & OSN & $I$ & $18.13 \pm 0.06$ & $0.173 \pm 0.01$ \\
\hline 3555 & 230 & OSN & $I$ & $18.2 \pm 0.06$ & $0.162 \pm 0.009$ \\
\hline 3808.7 & 240 & OSN & $I$ & $18.24 \pm 0.06$ & $0.156 \pm 0.009$ \\
\hline 4064.6 & 240 & OSN & $I$ & $18.2 \pm 0.06$ & $0.161 \pm 0.009$ \\
\hline 4313.5 & 210 & OSN & $I$ & $18.2 \pm 0.06$ & $0.163 \pm 0.009$ \\
\hline 4553.9 & 240 & OSN & $I$ & $18.31 \pm 0.06$ & $0.147 \pm 0.008$ \\
\hline 37.8 & 16 & BOOTES & $r^{\prime}$ & $16.14 \pm 0.15$ & $1.711 \pm 0.235$ \\
\hline 79.8 & 17 & BOOTES & $r^{\prime}$ & $16.48 \pm 0.14$ & $1.25 \pm 0.16$ \\
\hline 139.4 & 10 & LT & $R$ & $17.19 \pm 0.18$ & $0.545 \pm 0.088$ \\
\hline 149.6 & 10 & $\mathrm{LT}$ & $R$ & $16.73 \pm 0.12$ & $0.827 \pm 0.093$ \\
\hline
\end{tabular}


Table 4

(Continued)

\begin{tabular}{|c|c|c|c|c|c|}
\hline$t_{\text {mid }}(\mathrm{s})$ & $\operatorname{Exp}(\mathrm{s})$ & Telescope & Band & Magnitude & $F_{\nu}^{\mathrm{OPT}}(\mathrm{mJy})$ \\
\hline 159.8 & 10 & $\mathrm{LT}$ & $R$ & $16.82 \pm 0.15$ & $0.762 \pm 0.104$ \\
\hline 169.4 & 10 & LT & $R$ & $16.46 \pm 0.09$ & $1.056 \pm 0.09$ \\
\hline 179.6 & 10 & LT & $R$ & $16.66 \pm 0.12$ & $0.881 \pm 0.096$ \\
\hline 189.8 & 10 & $\mathrm{LT}$ & $R$ & $16.87 \pm 0.12$ & $0.73 \pm 0.082$ \\
\hline 199.4 & 10 & LT & $R$ & $16.79 \pm 0.12$ & $0.786 \pm 0.087$ \\
\hline 209.6 & 10 & LT & $R$ & $16.93 \pm 0.15$ & $0.693 \pm 0.094$ \\
\hline 219.2 & 10 & $\mathrm{LT}$ & $R$ & $17.09 \pm 0.19$ & $0.597 \pm 0.102$ \\
\hline 229.4 & 10 & LT & $R$ & $17.33 \pm 0.24$ & $0.483 \pm 0.105$ \\
\hline 249.2 & 10 & $\mathrm{LT}$ & $R$ & $17.02 \pm 0.15$ & $0.635 \pm 0.088$ \\
\hline 259.4 & 10 & $\mathrm{LT}$ & $R$ & $17 \pm 0.15$ & $0.648 \pm 0.089$ \\
\hline 269.6 & 10 & $\mathrm{LT}$ & $R$ & $17.09 \pm 0.14$ & $0.596 \pm 0.079$ \\
\hline 279.2 & 10 & $\mathrm{LT}$ & $R$ & $17.21 \pm 0.17$ & $0.534 \pm 0.083$ \\
\hline 289.4 & 10 & $\mathrm{LT}$ & $R$ & $17.08 \pm 0.16$ & $0.603 \pm 0.09$ \\
\hline 299 & 10 & LT & $R$ & $17.18 \pm 0.18$ & $0.549 \pm 0.092$ \\
\hline 334.1 & 60 & LT & $R$ & $17.5 \pm 0.11$ & $0.408 \pm 0.039$ \\
\hline 394.5 & 61 & $\mathrm{LT}$ & $R$ & $17.47 \pm 0.1$ & $0.418 \pm 0.038$ \\
\hline 454.7 & 60 & LT & $R$ & $17.49 \pm 0.11$ & $0.413 \pm 0.042$ \\
\hline 514.7 & 60 & LT & $R$ & $17.68 \pm 0.12$ & $0.345 \pm 0.037$ \\
\hline 574.1 & 60 & $\mathrm{LT}$ & $R$ & $17.78 \pm 0.12$ & $0.315 \pm 0.033$ \\
\hline 634.1 & 60 & LT & $R$ & $17.78 \pm 0.12$ & $0.314 \pm 0.035$ \\
\hline 692.9 & 57 & $\mathrm{LT}$ & $R$ & $18.19 \pm 0.16$ & $0.217 \pm 0.033$ \\
\hline 758.1 & 61 & $\mathrm{LT}$ & $R$ & $18.01 \pm 0.12$ & $0.254 \pm 0.029$ \\
\hline 818.3 & 60 & $\mathrm{LT}$ & $R$ & $18.04 \pm 0.14$ & $0.248 \pm 0.032$ \\
\hline 878.3 & 60 & $\mathrm{LT}$ & $R$ & $18.61 \pm 0.22$ & $0.148 \pm 0.029$ \\
\hline 938.3 & 60 & $\mathrm{LT}$ & $R$ & $18.21 \pm 0.16$ & $0.214 \pm 0.03$ \\
\hline 998.7 & 61 & $\mathrm{LT}$ & $R$ & $18.29 \pm 0.2$ & $0.199 \pm 0.036$ \\
\hline 1118.3 & 60 & $\mathrm{LT}$ & $R$ & $18.33 \pm 0.2$ & $0.191 \pm 0.034$ \\
\hline 1178.3 & 60 & $\mathrm{LT}$ & $R$ & $18.51 \pm 0.23$ & $0.164 \pm 0.034$ \\
\hline 1238.3 & 60 & $\mathrm{LT}$ & $R$ & $18.4 \pm 0.18$ & $0.18 \pm 0.029$ \\
\hline 1296.5 & 57 & LT & $R$ & $18.22 \pm 0.15$ & $0.211 \pm 0.029$ \\
\hline 1632.4 & 597 & $\mathrm{LT}$ & $R$ & $18.34 \pm 0.16$ & $0.188 \pm 0.027$ \\
\hline 2610 & 478 & $\mathrm{LT}$ & $R$ & $18.32 \pm 0.17$ & $0.193 \pm 0.029$ \\
\hline 3215.1 & 597 & $\mathrm{LT}$ & $R$ & $18.53 \pm 0.21$ & $0.159 \pm 0.03$ \\
\hline 306.2 & 60 & STELLA & $r^{\prime}$ & $17.33 \pm 0.06$ & $0.476 \pm 0.025$ \\
\hline 410.5 & 60 & STELLA & $r^{\prime}$ & $17.55 \pm 0.05$ & $0.386 \pm 0.019$ \\
\hline 514.9 & 60 & STELLA & $r^{\prime}$ & $17.78 \pm 0.05$ & $0.312 \pm 0.016$ \\
\hline 619.3 & 60 & STELLA & $r^{\prime}$ & $17.83 \pm 0.05$ & $0.299 \pm 0.015$ \\
\hline 723.7 & 60 & STELLA & $r^{\prime}$ & $18.05 \pm 0.06$ & $0.243 \pm 0.014$ \\
\hline 828.1 & 60 & STELLA & $r^{\prime}$ & $18.17 \pm 0.07$ & $0.218 \pm 0.013$ \\
\hline 932.3 & 60 & STELLA & $r^{\prime}$ & $18.28 \pm 0.09$ & $0.198 \pm 0.016$ \\
\hline 1088.8 & 120 & STELLA & $r^{\prime}$ & $18.35 \pm 0.08$ & $0.185 \pm 0.013$ \\
\hline 1297.5 & 120 & STELLA & $r^{\prime}$ & $18.28 \pm 0.07$ & $0.197 \pm 0.012$ \\
\hline 1506.3 & 120 & STELLA & $r^{\prime}$ & $18.35 \pm 0.08$ & $0.185 \pm 0.014$ \\
\hline 1714.9 & 120 & STELLA & $r^{\prime}$ & $18.29 \pm 0.09$ & $0.195 \pm 0.016$ \\
\hline 2001 & 30 & LT-IO:O* & $R$ & $18.4 \pm 0.1$ & $0.177 \pm 0.016$ \\
\hline 5240 & 100 & NOT$^{*}$ & $R$ & $18.78 \pm 0.02$ & $0.125 \pm 0.002$ \\
\hline 24516 & 600 & VATT* & $R$ & $20.07 \pm 0.03$ & $0.038 \pm 0.001$ \\
\hline 110916 & 1500 & VATT $*$ & $R$ & $21.7 \pm 0.1$ & $0.008 \pm 0.001$ \\
\hline 3004.3 & 10 & GTC & $r^{\prime}$ & $18.62 \pm 0.01$ & $0.173 \pm 0.002$ \\
\hline 3160.9 & 10 & GTC & $r^{\prime}$ & $18.62 \pm 0.01$ & $0.179 \pm 0.002$ \\
\hline 2305.2 & 300 & IAC & $R$ & $18.38 \pm 0.03$ & $0.179 \pm 0.004$ \\
\hline 129 & 11 & $\mathrm{LT}$ & $V$ & $18.2 \pm 0.25$ & $0.277 \pm 0.062$ \\
\hline 139.4 & 10 & LT & $V$ & $18 \pm 0.18$ & $0.331 \pm 0.055$ \\
\hline 149.6 & 10 & $\mathrm{LT}$ & $V$ & $17.54 \pm 0.11$ & $0.499 \pm 0.05$ \\
\hline 159.8 & 10 & $\mathrm{LT}$ & $V$ & $17.12 \pm 0.08$ & $0.732 \pm 0.05$ \\
\hline 169.4 & 10 & $\mathrm{LT}$ & $V$ & $17.11 \pm 0.07$ & $0.739 \pm 0.048$ \\
\hline 179.6 & 10 & $\mathrm{LT}$ & $V$ & $17.21 \pm 0.08$ & $0.677 \pm 0.052$ \\
\hline 189.8 & 10 & $\mathrm{LT}$ & $V$ & $17.21 \pm 0.08$ & $0.673 \pm 0.051$ \\
\hline 199.4 & 10 & $\mathrm{LT}$ & V & $17.53 \pm 0.1$ & $0.504 \pm 0.045$ \\
\hline 209.6 & 10 & $\mathrm{LT}$ & V & $17.72 \pm 0.12$ & $0.423 \pm 0.048$ \\
\hline 219.2 & 10 & $\mathrm{LT}$ & $V$ & $17.67 \pm 0.14$ & $0.444 \pm 0.059$ \\
\hline 229.4 & 10 & $\mathrm{LT}$ & $V$ & $17.53 \pm 0.12$ & $0.501 \pm 0.056$ \\
\hline 249.2 & 10 & $\mathrm{LT}$ & $V$ & $17.49 \pm 0.11$ & $0.52 \pm 0.052$ \\
\hline
\end{tabular}


Table 4

(Continued)

\begin{tabular}{|c|c|c|c|c|c|}
\hline$t_{\text {mid }}(\mathrm{s})$ & $\operatorname{Exp}(\mathrm{s})$ & Telescope & Band & Magnitude & $F_{\nu}^{\mathrm{OPT}}(\mathrm{mJy})$ \\
\hline 269 & 10 & $\mathrm{LT}$ & V & $17.77 \pm 0.11$ & $0.404 \pm 0.042$ \\
\hline 279.2 & 10 & LT & V & $17.84 \pm 0.14$ & $0.381 \pm 0.048$ \\
\hline 299 & 10 & $\mathrm{LT}$ & V & $17.73 \pm 0.11$ & $0.42 \pm 0.044$ \\
\hline 334.1 & 60 & $\mathrm{LT}$ & V & $17.82 \pm 0.07$ & $0.384 \pm 0.023$ \\
\hline 394.1 & 60 & $\mathrm{LT}$ & $V$ & $17.79 \pm 0.05$ & $0.393 \pm 0.019$ \\
\hline 574.1 & 60 & LT & V & $18.29 \pm 0.08$ & $0.249 \pm 0.018$ \\
\hline 634.1 & 60 & $\mathrm{LT}$ & $V$ & $18.53 \pm 0.1$ & $0.2 \pm 0.019$ \\
\hline 692.9 & 57 & $\mathrm{LT}$ & V & $18.55 \pm 0.11$ & $0.197 \pm 0.019$ \\
\hline 758.1 & 61 & $\mathrm{LT}$ & V & $18.34 \pm 0.08$ & $0.239 \pm 0.017$ \\
\hline 818.3 & 60 & $\mathrm{LT}$ & V & $18.86 \pm 0.14$ & $0.148 \pm 0.019$ \\
\hline 878.3 & 60 & LT & $V$ & $18.67 \pm 0.11$ & $0.176 \pm 0.017$ \\
\hline 1238.3 & 60 & LT & V & $18.81 \pm 0.1$ & $0.155 \pm 0.014$ \\
\hline 1296.5 & 57 & LT & V & $18.89 \pm 0.12$ & $0.144 \pm 0.015$ \\
\hline 1632.4 & 597 & LT & $V$ & $18.81 \pm 0.1$ & $0.155 \pm 0.015$ \\
\hline 2596.7 & 537 & $\mathrm{LT}$ & $V$ & $18.69 \pm 0.08$ & $0.172 \pm 0.013$ \\
\hline 3195.4 & 537 & $\mathrm{LT}$ & $V$ & $18.76 \pm 0.09$ & $0.161 \pm 0.013$ \\
\hline 1826.4 & 300 & IAC & $V$ & $18.81 \pm 0.03$ & $0.155 \pm 0.004$ \\
\hline 1647.6 & 300 & IAC & $B$ & $19.24 \pm 0.03$ & $0.135 \pm 0.004$ \\
\hline
\end{tabular}

Note. Magnitudes are not corrected for the Galactic extinction, while flux densities are. * indicates data obtained from GCN Circulars.

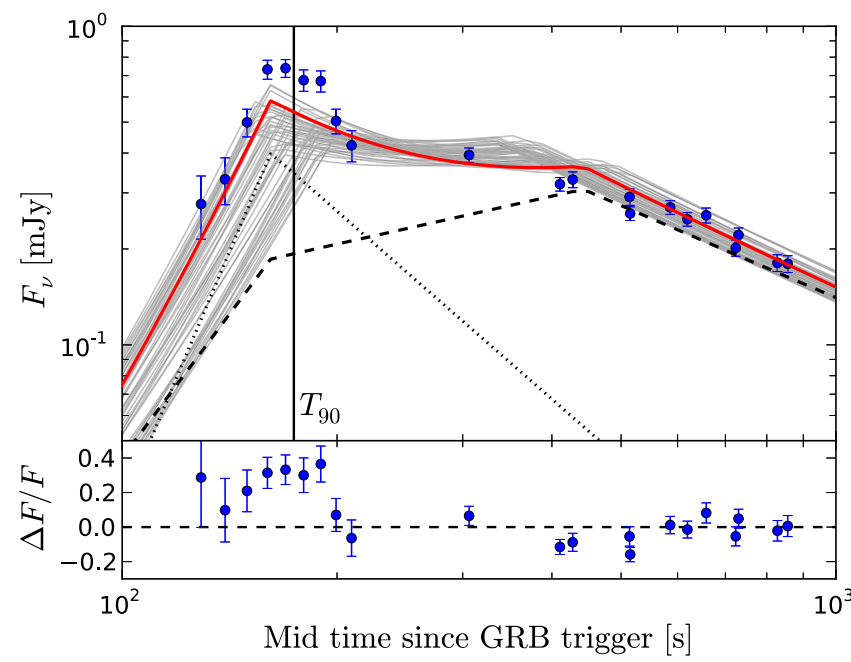

Figure 8. Top: combination of reverse-shock (dotted line) and forward-shock (dashed line) modeling for the thin-shell case and constant ISM, on a modified optical light curve (see Section 4.1.2). Gray solid lines represent simulated models (see Japelj et al. 2014), while the best model is depicted with the red solid line. The solid vertical line indicates $T_{90}$. Bottom: residuals between data points and the best model.

Moreover, the origin of early optical peaks is not consistent with density fluctuations in the circumburst medium-changes in temporal slopes during the optical flares would require prohibitively high density fluctuations to explain them (Nakar $\&$ Granot 2007). In summary, we therefore favor an internal shock origin for the early optical flares, as described in Section 4.1.1.

\subsection{Post-flaring Afterglow}

After the initial flaring episode (after $300 \mathrm{~s}$ ), the optical emission shows decay with power-law index $\alpha_{\text {decay }} \approx 1$, which is consistent with the forward-shock afterglow origin. This is also supported by the late time broadband SED, which is best modeled using a broken power-law (see Section 3.3), with the difference of spectral indices $\Delta \beta \approx 0.5$, as expected for a cooling break (Sari et al. 1998). Due to a cooling frequency lying between optical and X-ray, one would expect that X-ray LC would decay steeper than optical, but this is not evident at late time, when X-ray and optical decay indices are comparable. The contradiction can be explained if the surrounding environment is not a constant ISM medium, but stratified medium with circumburst medium density given by $n=A R^{-k}$ (Chevalier \& Li 2000). In this case, temporal decay indices for forward shock emission are given by $\alpha_{\text {decay }}=-(3 p(4-k)+5 k-12) /(4(4-k))$ and $\alpha_{\text {decay }}=-(3 p-2) / 4$, before and after the cooling break, respectively. Similar temporal decay indices from different spectral regimes are thus possible in environments with $k \sim 4 / 3$.

\subsection{Late Re-brightening: Energy Injection}

At $\sim 2000 \mathrm{~s}$, the optical LC shows a transition from a powerlaw decline with $\alpha_{\text {decay }} \sim 1$ to a plateau phase, followed by a power-law decline with $\alpha_{\text {decay }} \sim 0.8$ (Table 2). Consistent power-law decline after the plateau phase is also obtained from the X-ray LC. This could be explained in the context of late time continued energy injection by a central engine (e.g., Rees \& Mészáros 1998; Zhang \& Mészáros 2002; Zhang et al. 
2006). A shallower decay index after the plateau phase implies gradual and continuous energy injection. Following Sari \& Mészáros (2000), we can estimate that the change in the optical decay slope of the forward shock corresponds to the change in the power-law exponent of the ejected mass distribution $\left(M(>\gamma) \propto \gamma^{-s}\right)$ from $s \approx 1$ (instantaneous case) to $s \sim 2$, which is typical for moderate continuous energy injection.

Density bumps or voids in the surrounding medium can produce bumps in the afterglow LCs (Uhm \& Zhang 2014), but the decay index is expected to return to the same value as before the peak, which is not the case in this GRB (the difference is $\sim 0.2$ ). Similarly, multi-component jets, together with a possibility that an outflow is seen slightly off-axis, can produce LCs with additional re-brightening (Kumar \& Piran 2000), but if the circumburst environment stays of the same type, the LC behavior before and after the re-brightening should be similar.

\subsection{Optical Polarization Upper Limits}

GRB polarimetry offers a direct probe of the structure of magnetic fields within the emission region. Late-time optical measurements taken hours to days after the burst show low values of linear polarization of a few percent, consistent with synchrotron emission from a tangled magnetic field in the shocked ISM (e.g., Greiner et al. 2003; Lazzati et al. 2003; Wiersema et al. 2012, 2014). Circular polarization was recently detected at the $0.6 \%$ level, which although small, is larger than expected theoretically (Wiersema et al. 2014). In contrast, measurements of the early afterglow, taken hundreds of seconds after the burst, when the properties of the original fireball are still encoded in the emitted light, can show high linear polarizations up to $30 \%$, particularly when a reverse shock can be identified in the LC - this is consistent with theoretical predictions for a large-scale ordered magnetic flow advected from the central engine (Steele et al. 2009; Uehara et al. 2012; Mundell et al. 2013).

High degrees of gamma-ray polarization $(P \sim 40 \%-60 \%)$ have been measured during the prompt emission for a number of GRBs, using dedicated instruments on INTEGRAL and IKAROS satellites (e.g., Götz et al. 2009, 2013, 2014; Yonetoku et al. 2012), despite the highly variable and peaked nature of prompt flares. The origin of the prompt emission is still unknown but these polarization levels suggest that magnetic fields play an important role. However, because gamma-ray polarization measurements are difficult to obtain, and often accompanied by large systematic uncertainties, the measurement of optical polarization at early times would provide additional constraints on the origin of the prompt emission.

GRB 140430A provides a unique opportunity, as its optical emission was detected during the ongoing prompt emission. The upper limits we derive on linear optical polarization in the very early afterglow (see Section 3.4) are relatively low at $P<$ $22 \%-30 \%(1 \sigma)$ or $P<22 \%-30 \%(3 \sigma)$, during the first optical flare. The polarization limit of $P \lesssim 20 \%(1 \sigma)$ during the second optical flare is less constrained due to $\mathrm{S} / \mathrm{N}$ limitations. If early time optical flares originate from within the internal shock region in the jet and if the jet is threaded with large-scale and ordered magnetic fields, we would expect the emission to be highly polarized (up to $60 \%$, depending on electron distribution). In contrast, the observed polarization could be reduced in a number of scenarios if (a) the magnetic field is tangled locally, (b) there exists a number of patches within a region of size $1 / \Gamma$ where the magnetic field is ordered locally but our viewing angle at the time of observation is already larger due to smaller $\Gamma$ (e.g., Gruzinov \& Waxman 1999; Granot \& Königl 2003; Lyutikov et al. 2003), (c) there are multiple, unresolved but highly variable optical components that average temporally to a lower net value of polarization, or (d) emission from spatially distinct regions-such as internal shock and the external reverse shock regions (see discussion in Section 4.1) each with high polarization but different position angles are observed simultaneously, resulting in an apparent lower total polarization degree.

In summary, the total degree of early time optical polarization for GRB 140430A measured during the highenergy X-ray and gamma-ray flares is lower than the commonly obtained levels of prompt gamma-ray polarization measurements of other GRBs and lower than that attributed to reverse-shock emission in GRB 120308A (Mundell et al. 2013). As described above, the intrinsic polarization of this GRB may be low or the vector averaging of different highly polarized components may explain the lower net observed polarization. Definitive interpretations of prompt gamma-ray emission will be possible when measurements of both prompt gamma-ray polarization and contemporaneous optical polarization are available for comparison in the same GRB.

\section{CONCLUSIONS}

In this paper, we present a detailed analysis of the early optical emission from GRB 140430A, which was observed with the fast polarimeter RINGO3 mounted on the Liverpool Telescope. Due to the fast response of the instrumentation, we were able to obtain optical measurements in three color bands during the ongoing prompt gamma-ray emission and X-ray flaring episode. Our measurements and analysis show the following.

1. The multi-color optical LC at early times is complex, and best described by two optical flares superimposed on a broad underlying afterglow component. The optical flares temporally coincident with the high-energy X-ray and gamma-ray flares (prompt GRB emission), suggesting a central engine origin. Temporal and spectral analysis of the early time broadband data set indicates emission from internal shock dissipation, dominating over external shock afterglow.

2. At $\sim 2000 \mathrm{~s}$, a late time re-brightening (plateau) occurs, which is interpreted as being due to late central engine activity (reactivation) with continuous energy injection, based on shallower post-plateau decay. Structured jet and density fluctuations in the circumburst medium are disfavored due to change in decay slope and due to no spectral variability.

3. Optical polarimetry in three bands during the ongoing prompt emission showed that contemporaneous optical flares are not highly polarized, with the upper limits as low as $P<12 \%(1 \sigma)$. Alternatively, time-averaging of multiple emission components (to obtain sufficient $\mathrm{S} / \mathrm{N}$ for polarimetry) with different polarization behavior may be responsible for lowering the overall polarization estimate.

Time-resolved polarimetry during the prompt gamma-ray phase is vital to determine whether individual emission 
components in early optical LCs are polarized. Ultimately, observing the same GRBs simultaneously in optical and gamma-ray polarization will revolutionize our understanding of GRB emission mechanisms.

We thank the anonymous referee for valuable comments that improved the paper. D.K. acknowledges support from the Science and Technology Facilities Council (STFC). C.G.M. acknowledges support from the Royal Society, the Wolfson Society, and STFC. A.J.C.T. thanks the support of the Spanish Ministry Project AYA2012-39727-C03-01 and excellent support from the OSN staff. The Liverpool Telescope is operated on the island of La Palma by Liverpool John Moores University in the Spanish Observatorio del Roque de los Muchachos of the Instituto de Astrofisica de Canarias with financial support from the UK Science and Technology Facilities Council. The Gran Telescopio Canarias (GTC) is installed in the Spanish Observatorio del Roque de los Muchachos of the Instituto de Astrofisica de Canarias, in the island of La Palma. This work made use of data obtained with the STELLA robotic telescopes in Tenerife, an AIP facility jointly operated by AIP and IAC. This work made use of data supplied by the UK Swift Science Data Centre at the University of Leicester. The Swift mission is funded in the UK by STFC, in Italy by ASI, and in the USA by NASA.

\section{REFERENCES}

Akerlof, C., et al. 1999, Natur, 398, 400

Arnold, D. M., Steele, I. A., Bates, S. D., Mottram, C. J., \& Smith, R. J. 2012, Proc. SPIE, 8446, 2

Axelsson, M., \& Borgonovo, L. 2015, MNRAS, 447, 3150

Barthelmy, S. D., Barbier, L. M., Cummings, J. R., et al. 2005, SSRv, 120, 143 Beloborodov, A. M. 2005, ApJ, 618, 13

Beloborodov, A. M., Daigne, F., Mochkovitch, R., \& Uhm, Z. L. 2011, MNRAS, 410, 2422

Beniamini, P., Nava, L., Duran, R. B., \& Piran, T. 2015, MNRAS, 454, 1073 Bernardini, M. G., Margutti, R., Guidorzi, C., \& Mao, J. 2011, A\&A, 526, 27 Beuermann, K., Hessman, F. V., Reinsch, K., et al. 1999, A\&A, 352, L26

Blake, C. H., Bloom, J. S., Starr, D. L., et al. 2005, Natur, 435, 181

Boër, M., Atteia, J. L., Damerdji, Y., et al. 2006, ApJL, 638, L71

Breeveld, A. A., \& Siegel, M. H. 2014, GCN, 16198

Burrows, D. N., Romano, P., Falcone, A., et al. 2005, Sci, 309, 1833

Burrows, D. N., Hill, J. E., Nousek, J. A., et al. 2005, SSRv, 120, 165

Butler, N. R., \& Kocevski, D. 2007, ApJ, 663, 407

Castro-Tirado, A. J., Jelínek, M., Gorosabel, J., et al. 2012, ASInC, 7, 313

Cepa, J., Aguiar, M., Escalera, V. G., et al. 2000, Proc. SPIE, 4008, 623

Chevalier, R. A., \& Li, Z. Y. 2000, ApJ, 536, 195

Chincarini, G., Moretti, A., Romano, P., et al. 2007, ApJ, 671, 1903

Chincarini, G., Mao, J., Margutti, R., et al. 2010, MNRAS, 406, 2113

Clarke, D., \& Neumayer, D. 2002, A\&A, 383, 360

Cucchiara, A., Levan, A. J., Fox, D. B., et al. 2011, ApJ, 736, 7

de Pasquale, M., Kuin, N. P. M., Oates, S., et al. 2015, MNRAS, 449, 1024

Elliott, J., Yu, H.-F., Schmidl, S., et al. 2014, A\&A, 562, 100

Evans, P. A., Beardmore, A. P., Page, K. L., et al. 2009, MNRAS, 397, 1177

Evans, P. A., Willingale, R., Osborne, J. P., et al. 2010, A\&A, 519, A102

Evans, P. A., Goad, M. R., Osborne, J. P., \& Beardmore, A. P. 2014, GCN, 16195

Fukugita, M., Ichikawa, T., Gunn, J. E., et al. 1996, AJ, 111, 1748

Gehrels, N., Chincarini, G., Giommi, P., et al. 2004, ApJ, 611, 1005

Gendre, B., Atteia, J. L., Boër, M., et al. 2012, ApJ, 748, 59

Gendre, B., Stratta, G., Atteia, J. L., et al. 2013, ApJ, 766, 30

Giannios, D. 2006, A\&A, 455, L5

Golyandina, N., Korobeynikov, A., Shlemov, A., \& Usevichet, K. 2015, J Stat Soft, 6721

Gomboc, A. 2012, ConPh, 53, 339

Gomboc, A., Kobayashi, S., Guidorzi, C., et al. 2008, ApJ, 687, 443

Gomboc, A., Kobayashi, S., Mundell, C. G., et al. 2009, AIPC, 1133, 145
Gomboc, A., et al. 2015, in preparation

Gorosabel, J., Gomez, E., Gonzalez-Morales, P. A., \& Cepa, J. 2014, GCN, 16205

Götz, D., Laurent, P., Lebrun, F., Daigne, F., \& Bošnjak, Ž. 2009, ApJ, 695, 208

Götz, D., Covino, S., Fernández-Soto, A., Laurent, P., \& Bošnjak, Ž. 2013, MNRAS, 431, 3550

Götz, D., Laurent, P., Antier, S., et al. 2014, MNRAS, 444, 2776

Granot, J., \& Königl, A. 2003, ApJ, 594, 83

Greiner, J., Klose, S., Reinsch, K., et al. 2003, Natur, 426, 157

Greiner, J., Krühler, T., McBreen, S., et al. 2009, ApJ, 693, 1912

Greiner, J., Yu, H.-F., Krühler, T., et al. 2014, A\&A, 568, 75

Gruzinov, A., \& Waxman, E. 1999, ApJ, 511, 852

Guidorzi, C., Yu, H.-F., Krühler, T., et al. 2006, PASP, 118, 288

Guidorzi, C., Monfardini, A., Gomboc, A., et al. 2009, A\&A, 499, 439

Guidorzi, C., Clemens, C., Kobayashi, S., et al. 2011, MNRAS, 417, 2124

Guidorzi, C., Mundell, C. G., Harrison, R., et al. 2015a, MNRAS, 438, 752

Guidorzi, C., Dichiara, S., Frontera, F., et al. 2015b, ApJ, 801, 57

Ioka, K., Kobayashi, S., \& Zhang, B. 2005, ApJ, 631, 429

Japelj, J., Kopač, D., Kobayashi, S., et al. 2014, ApJ, 785, 84

Japelj, J., Covino, S., Gomboc, A., et al. 2015, A\&A, 579, 74

King, O. G., Blinov, D., Giannios, D., et al. 2014, MNRAS, 445, L114

Klebesadel, R. W., Strong, I. B., \& Olson, R. A. 1973, ApJL, 182, L85

Kobayashi, S. 2000, ApJ, 545, 807

Kobayashi, S., Piran, T., \& Sari, R. 1997, ApJ, 490, 92

Kobayashi, S., \& Zhang, B. 2003, ApJ, 597, 455

Kopač, D., Kobayashi, S., Gomboc, A., et al. 2013, ApJ, 772, 73

Krimm, H. A., Barthelmy, S. D., Baumgartner, W. H., et al. 2014, GCN, 16200

Krühler, T., Greiner, J., \& McBreen, S. 2009, ApJ, 697, 758

Krühler, T., Malesani, D., de Ugarte Postigo, A., Melandri, A., \& Fynbo, J. P. U. 2014, GCN, 16194

Kumar, P., \& Panaitescu, A. 2008, MNRAS, 391, L19

Kumar, P., \& Piran, T. 2000, ApJ, 535, 152

Lazzati, D., Covino, S., di Serego Alighieri, S., et al. 2003, A\&A, 410, 823 Li, Z., \& Waxman, E. 2008, ApJ, 674, 65

Lu, R.-J., Wei, J.-J., Liang, E.-W., et al. 2012, ApJ, 756, 112

Lyutikov, M. 2006, NJPh, 8, 119

Lyutikov, M., Pariev, V. I., \& Blandford, R. D. 2003, ApJ, 597, 998

Margutti, R., Guidorzi, C., Chincarini, G., et al. 2010, MNRAS, 406, 2149

Margutti, R., Chincarini, G., Granot, J., et al. 2011, MNRAS, 417, 2144

Melandri, A., Mundell, C. G., Kobayashi, S., et al. 2008, ApJ, 686, 1209

Melandri, A., Kobayashi, S., Mundell, C. G., et al. 2010, ApJ, 723, 1331

Melandri, A., Guidorzi, C., Gomboc, A., Smith, R. J., \& Mundell, C. G. 2014, GCN, 16192

Mészáros, P. 2006, RPPh, 69, 2259

Mészáros, P., \& Rees, M. J. 1997, ApJ, 476, 232

Monfardini, A., Kobayashi, S., Giudorzi, C., et al. 2006, ApJ, 648, 1125

Mundell, C. G., Steele, I. A., Smith, R. J., et al. 2007a, Sci, 315, 1822

Mundell, C. G., Melandri, A., Guidorzi, C., et al. 2007b, ApJ, 660, 489

Mundell, C. G., Kopač, D., Arnold, D. M., et al. 2013, Natur, 504, 119

Nakar, E., \& Granot, J. 2007, MNRAS, 380, 1744

Nappo, F., Ghisellini, G., Ghirlanda, G., et al. 2014, MNRAS, 445, 1625

Norris, J. P., Bonnell, J. T., Kazanas, D., et al. 2005, ApJ, 627, 324

Nousek, J. A., Kouveliotou, C., Grupe, D., et al. 2006, ApJ, 642, 389

O'Brien, P. T., Willingale, R., Osborne, J., et al. 2006, ApJ, 647, 1213

Page, K. L., Willingale, R., Osborne, J. P., et al. 2007, ApJ, 663, 1125

Panaitescu, A. 2008, MNRAS, submitted (arXiv:0811.1235)

Panaitescu, A., \& Vestrand, W. T. 2008, MNRAS, 387, 497

Pei, Y. C. 1992, ApJ, 395, 130

Piran, T. 1999, PhR, 314, 575

Planck Collaboration, 2014, A\&A, 571, 16

Racusin, J. L., Karpov, S. V., Sokolowski, M., et al. 2008, Natur, 445, 183

Rees, M. J., \& Mészáros, P. 1992, MNRAS, 258, 41

Rees, M. J., \& Mészáros, P. 1994, ApJ, 430, 93

Rees, M. J., \& Mészáros, P. 1998, ApJ, 496, 1

Rees, M. J., \& Mészáros, P. 2005, ApJ, 628, 847

Roming, P. W. A., Kennedy, T. E., Mason, K. O., et al. 2005, SSRv, 120, 95

Rossi, A., Schulze, S., Klose, S., et al. 2011, A\&A, 529, 142

Sakamoto, T., Barthelmy, S. D., Baumgartner, W.H., et al. 2011, ApJS, 195, 2 Salvaterra, R., Della Valle, M., Campana, S., et al. 2009, Natur, 461, 1258

Sari, R., Piran, T., \& Narayan, R. 1998, ApJL, 497, L17

Sari, R., \& Mészáros, P. 2000, ApJ, 535, 33

Schlafly, E. F., \& Finkbeiner, D. P. 2011, ApJ, 737, 103

Siegel, M. H., Baumgartner, W. H., Chester, M. M., et al. 2014, GCN, 16190 
Steele, I. A., Smith, R. J., Rees, P. C., et al. 2004, Proc. SPIE, 5489, 679 Steele, I. A., Mundell, C. G., Smith, R. J., Kobayashi, S., \& Guidorzi, C. 2009, Natur, 462, 767

Tanvir, N. R., Fox, D. B., Levan, A. J., et al. 2009, Natur, 461, 1254

Thöne, C. C., Kann, D. A., Jóhannesson, G., et al. 2010, A\&A, 523, A70

Troja, E., Piro, L., Vasileiou, V., et al. 2015, ApJ, 803, 10

Uehara, T., Toma, K., Kawabata, K. S., et al. 2012, ApJ, 752, 6

Uhm, Z. L., \& Zhang, B. 2014, ApJ, 789, 39

Vedrenne, G., \& Atteia, J.-L. 2009, Gamma-Ray Bursts: The Brightest Explosions in the Universe (Berlin: Springer)

Vestrand, W. T., Wozniak, P. R., Wren, J. A., et al. 2005, Natur, 435, 178

Vestrand, W. T., Wren, J. A., Panaitescu, A., et al. 2014, Sci, 343, 38

Virgili, F. J., Mundell, C., Palshin, V., et al. 2013, ApJ, 778, 54
Wiersema, K., Curran, T., Krühler, T., et al. 2012, MNRAS, 426, 2

Wiersema, K., Covino, S., Toma, K., et al. 2014, Natur, 509, 201

Willingale, R., Starling, R. L. C., Beardmore, A. P., Tanvir, N. R., \& O'Brien, P. T. 2013, MNRAS, 431, 394

Yonetoku, D., Murakami, T., Gunji, S., et al. 2012, ApJ, 758, 1

Yost, S. A., Swan, H. F., Rykoff, E. S., et al. 2007a, ApJ, 657, 925

Yost, S. A., Aharonian, F., Akerlof, C. W., et al. 2007b, ApJ, 669, 1107

Zhang, B., \& Mészáros, P. 2002, ApJ, 566, 712

Zhang, B., \& Yan, H. 2011, ApJ, 726, 90

Zhang, B., Kobayashi, S., \& Mészáros, P. 2003, ApJ, 595, 950

Zhang, B., Fan, Y. Z., Dyks, J., et al. 2006, ApJ, 642, 354

Zhang, B.-B., Liang, E.-W., \& Zhang, B. 2007, ApJ, 666, 1002

Zheng, W., Shen, R. F., Sakamoto, T., et al. 2012, ApJ, 751, 90 